ACCePted by ApJ, March 04, 2013

Preprint typeset using $\mathrm{IAT}_{\mathrm{E} X} \mathrm{X}$ style emulateapj v. 5/2/11

\title{
PLASMOID EJECTIONS AND LOOP CONTRACTIONS IN AN ERUPTIVE M7.7 SOLAR FLARE: EVIDENCE OF PARTICLE ACCELERATION AND HEATING IN MAGNETIC RECONNECTION OUTFLOWS
}

\author{
Wei Liu ${ }^{1,2}$, Qingrong Chen ${ }^{3}$, Vahé Petrosian ${ }^{3}$ \\ Accepted by ApJ, March 04, 2013
}

\begin{abstract}
Where particle acceleration and plasma heating take place in relation to magnetic reconnection is a fundamental question for solar flares. We report analysis of an M7.7 flare on 2012 July 19 observed by SDO/AIA and RHESSI. Bi-directional outflows in forms of plasmoid ejections and contracting cuspshaped loops originate between an erupting flux rope and underlying flare loops at speeds of typically $200-300 \mathrm{~km} \mathrm{~s}^{-1}$ up to $1050 \mathrm{~km} \mathrm{~s}^{-1}$. These outflows are associated with spatially separated double coronal X-ray sources with centroid separation decreasing with energy. The highest temperature is located near the nonthermal X-ray loop-top source well below the original heights of contracting cusps near the inferred reconnection site. These observations suggest that the primary loci of particle acceleration and plasma heating are in the reconnection outflow regions, rather than the reconnection site itself. In addition, there is an initial ascent of the X-ray and EUV loop-top source prior to its recently recognized descent, which we ascribe to the interplay among multiple processes including the upward development of reconnection and the downward contractions of reconnected loops. The impulsive phase onset is delayed by 10 minutes from the start of the descent, but coincides with the rapid speed increases of the upward plasmoids, the individual loop shrinkages, and the overall loop-top descent, suggestive of an intimate relation of the energy release rate and reconnection outflow speed. Subject headings: acceleration of particles - Sun: flares - Sun: UV radiation — Sun: X-rays, gamma rays
\end{abstract}

\section{INTRODUCTION}

Magnetic reconnection is believed to be the primary energy release mechanism during solar flares, but where and how the released energy is transformed to heat the plasma and accelerate particles remain unclear (for reviews, see Holman et al. 2011; Fletcher et al. 2011; Petrosian 2012; Ravmond et al. 2012). Evidence of magnetic reconnection and current sheets on the Sun has been observed in various situations and wavelengths.

A major advance in the last decade was the discovery of a second coronal source above a commonly observed loop-top source in X-rays and radio wavelengths (Sui \& Holman 2003; Sui et al. 2004; Pick et al. 2005 Veronig et al. 2006; Li \& Gan 2007; Liu et al. 2008, 2009c; Chen \& Petrosian 2012; Su et al.|2012; Bain et al. 2012; Glesener et al. 2012). Such double sources often exhibit higher-energy emission being closer to each other, indicating higher temperatures or harder spectra of accelerated electrons in the inner region nearer to the presumable magnetic reconnection site.

Another surprise has been the descent of the loop-top X-ray source at typically $10-40 \mathrm{~km} \mathrm{~s}^{-1}$ early in the impulsive phase before its common ascent through the decay phase (Sui \& Holman 2003; Sui et al. 2004; Liu et al. 2004; Shen et al. 2008). The upper coronal source, however, usually keeps ascending all the time.

Shrinkages of entire flare loops (Svestka et al. 1987) at speeds on the order of $10 \mathrm{~km} \mathrm{~s}^{-1}$ have been ob-

\footnotetext{
${ }^{1}$ Lockheed Martin Solar and Astrophysics Laboratory, Building 252, 3251 Hanover Street, Palo Alto, CA 94304

2 W. W. Hansen Experimental Physics Laboratory, Stanford University, Stanford, CA 94305

${ }^{3}$ Department of Physics, Stanford University, Stanford, CA 94305
}

served in soft X-rays (SXRs; Forbes \& Acton 1996; Reeves et al. 2008), extreme ultraviolet (EUV; Li \& Gan 2006), and microwaves (Li \& Gan 2005; Reznikova et al. 2010). They were interpreted as contractions of newly reconnected loops due to magnetic tension as they evolve from initially cusp shapes toward more relaxed round shapes.

Often after the impulsive phase, bright loops and dark voids seen in SXR or EUV descend onto a flare arcade from above at greater speeds of typically $150 \mathrm{~km} \mathrm{~s}^{-1}$ (McKenzie \& Hudson 1999; Savage \& McKenzie 2011). At even greater heights of a few $R_{\odot}$, similar descending loops are seen in white-light coronagraphs, usually hours after a coronal mass ejection (CME; Wang et al. 1999; Sheeley et al. 2004). These features are also interpreted as contracting post-reconnection loops, but their observed speeds are only a small fraction of the expected coronal Alfvén speed on the order of $\sim 1000 \mathrm{~km} \mathrm{~s}^{-1}$.

Imaging and Doppler observations have also revealed bi-directional magnetic reconnection inflows (Yokovama et al. 2001; Milligan et al. 2010; Liu et al. 2010), outflows (Innes et al. 1997; Ko et al. 2003; Wang et al. 2007; Nishizuka et al. 2010; Hara et al. 2011; Watanabe et al. 2012), or both (Lin et al. 2005; Takasao et al. 2012; Savage et al. 2012).

The physics behind descending X-ray loop-top sources and their relationship with slow loop shrinkages, fast supra-arcade loop contractions, and reconnection outflows remain unclear, although some models have been proposed (e.g., Somov \& Kosugi 1997; Karlický \& Kosugi 2004). To fill this gap, we present here observations of a recent eruptive M7.7 flare from the Reuven Ramaty High Energy Solar Spectroscopic Imager (RHESSI; Lin et al. 2002) and Solar Dynamics Ob- 
servatory Atmospheric Imaging Assembly (SDO/AIA; Lemen et al. 2012). We find in this flare all the above interrelated phenomena, which can be understood in a unified picture as contractions of post-reconnection loops modulated by the interplay between energy release and cooling. The observed highest speed of $\sim 1000 \mathrm{~km} \mathrm{~s}^{-1}$ of loop contractions is comparable to the expected Alfvén speed. The maximum temperature and nonthermal looptop emission being away from the inferred reconnection site suggest that primary heating and particle acceleration take place in the outflow regions, rather than the reconnection site itself.

After an observational overview in Section 2, we present motions of the overall X-ray and EUV emission in Section 3. We examine bi-directional outflows in forms of plasmoids and contracting loops in Section 4. In Section 5. we analyze the spatial distribution of energy and temperature dependent emission, including double coronal X-ray sources. We conclude in Section 6, followed by two appendixes on supplementary AIA and STEREO observations.

TABLE 1

Event Time Line (2012 July 18-19)

\begin{tabular}{ll}
\hline $22: 18,07 / 18$ & Peak of the earlier C4.5 flare \\
$04: 17,07 / 19$ & Onset of the M7.7 flare and initial ascent \\
& of the overall X-ray and EUV loop-top source \\
\hline $05: 02-05: 07$ & $\begin{array}{l}\text { Onset of overall X-ray and EUV loop-top descent } \\
\text { and transition from slow to fast rise of the flux rope CME }\end{array}$ \\
& Max. velocity $\left(1050 \mathrm{~km} \mathrm{~s}^{-1}\right)$ of upward ejections \\
$05: 15$ & Max. velocity $\left(-58 \mathrm{~km} \mathrm{~s}^{-1}\right)$ of downward loop shrinkages \\
$05: 16$ & Max. velocity $\left(-7\right.$ to $\left.-23 \mathrm{~km} \mathrm{~s}{ }^{-1}\right)$ of overall \\
$05: 15-05: 20$ & X-ray and EUV loop-top descent \\
$05: 16-05: 43$ & Flare impulsive phase, hard X-ray burst \\
$05: 21-05: 31$ & $\begin{array}{l}\text { Min. height of overall X-ray and EUV loop-top descent } \\
\text { and onset of the second ascent }\end{array}$ \\
\hline $04: 17-05: 20$ & Upward ejections; downward, low-altitude fast contractions \\
$05: 20-16: 00$ & Downward, high-altitude fast loop contractions \\
$04: 17-16: 00$ & $\begin{array}{l}\text { Downward slow loop shrinkages } \\
\left.\text { Max. velocity (-918 km s } \mathrm{s}^{-1}\right) \text { of downward fast contractions }\end{array}$ \\
$06: 52$ &
\end{tabular}

\section{OVERVIEW OF OBSERVATIONS}

The event under study was an M7.7 flare that occurred at $\sim 4: 17$ UT on 2012 July 19 in NOAA active region (AR) 11520 on the southwest limb. It was well observed by RHESSI and SDO/AIA, but it was not detected by Fermi and its impulsive phase was missed by the X-ray Telescope on Hinode. Table 1 summarizes the event time line that will be discussed in detail.

Figure 1 shows the history of the flare emission. The GOES 1-8 $\AA$ flux peaks at 05:58 UT followed by a slow decay lasting almost one day. We define the interval of 05:16-05:43 UT as the impulsive phase, as marked by the two vertical dashed lines, which starts at the sudden rise of the RHESSI 25-50 keV flux and ends (during RHESSI night) when the time derivative of the GOES 1-8 $\AA$ flux drops to its level at the impulsive phase onset, assuming the Neupert (1968) effect at work. We call the intervals before and after the impulsive phase the pre-impulsive and decay phases. RHESSI has good coverage except for the late impulsive and early decay phases.

Figure 2 and its associated Movie A show AIA images of the event. An earlier C4.5 flare occurred in the same location, peaking at 22:18 UT on 2012 July 18 (see Figure 1(a)). This is a confined flare that produces a hot

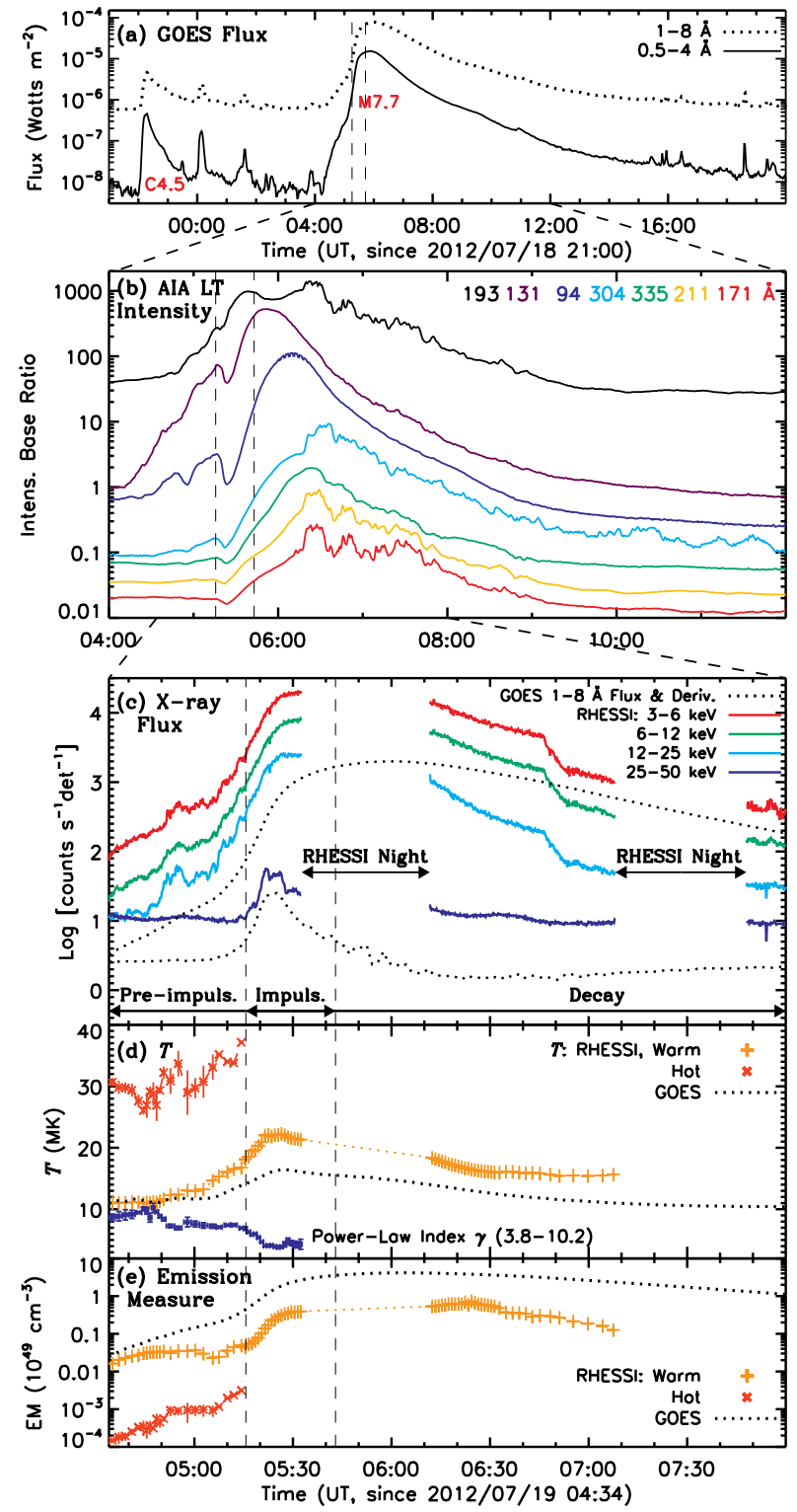

FIG. 1.- History of X-ray and EUV flare emission. (a) GOES SXR fluxes showing the preceding C4.5 flare and the main M7.7 flare. (b) $S D O /$ AIA intensity of the loop-top region (at projected height $h_{\text {ref }}=60^{\prime \prime}$ on Cut 0 , as shown in Figure [13) normalized by its initial value (base ratio). Color-coded for AIA channels, the curves are vertically shifted to avoid overlap and arranged from the top to bottom approximately in the temporal order of their initial response to the flare (in progressively cooler channels). (c) RHESSI count rates in colored solid lines and GOES 1-8 $\AA$ flux and its time derivative in black dotted lines, arbitrarily shifted vertically. The two vertical dashed lines indicate the impulsive phase. (d) Temperature and (e) emission measure of the flare plasma inferred from RHESSI (orange/red) and GOES (black) spectral fits, together with the power-law index $\gamma$ of the nonthermal component shown in blue in (d).

flux rope failing to erupt and cusp-shaped flare loops underneath it (Figure 2(a)). This configuration then gradually evolves for hours and finally becomes unstable, initiating the later, eruptive M7.7 flare, when the flux rope is expelled as a fast CME of $>1000 \mathrm{kms}^{-1}$. The flux rope in this two-stage eruption was reported by Patsourakos et al. (2013).

As shown in Figure 2(i), the trailing edge of the CME displays a clear "V-shape", which, together with the 
underlying "inverted-V shape" of cusp-like flare loops, suggests two Y-type null points with a vertical current sheet formed in between, as predicted in the classical picture of eruptive flares (Carmichael 1964; Sturrock 1966; Hirayama 1974; Kopp \& Pneuman 1976). Not predicted in that picture is the initial upward growth of the cusp followed by its rapid downward shrinkage around the early impulsive phase, prior to another, expected upward growth through the decay phase in a commonly observed candle-flame shape (Figures 2(a)-(1)). The initial growth and shrinkage are accompanied by the gradual rise and impulsive eruption of the overlying flux rope, respectively. Equally interesting are high-speed bidirectional outflows involving upward-moving plasmoids and downward-contracting pointed cusps (Figure 2, bottom: Figure 5). We examine these and related features observed by $R H E S S I$ and AIA in next several sections.

\section{OVERALL X-RAY AND EUV SOURCE MOTIONS}

We first follow the evolution of the morphologies and positions of overall X-ray and EUV sources.

\subsection{X-ray Source Morphology}

We reconstructed RHESSI X-ray images in energy bands from 3 to $50 \mathrm{keV}$ using the CLEAN algorithm and detectors 3-9. Depending on the count rate, we chose variable integration time ranging from $20 \mathrm{~s}$ during the impulsive phase to 4 minutes during the decay phase.

Figure 3 and its accompanying Movie show examples of RHESSI contours overlaid on AIA images. There is a persistent loop-top source at 6-10 keV (green) near the apex of the cusp-shaped EUV loops. Accompanying the evolution of the EUV loops mentioned above, this loop-top source undergoes a gradual ascent followed by a descent and then another ascent. This can be best seen in the last panel showing the temporal migration of the emission centroid obtained from a contour at $70 \%$ of the maximum of each image. Early descents of loop-top Xray sources have been recognized (e.g., Sui et al. 2004), but this is the first time that an evident preceding ascent is observed.

Early in the event there is an additional, weaker coronal X-ray source (panel (b)) located in the lower portion of the overlying flux rope. It later falls below detection with the eruption of the flux rope. During the impulsive phase (panel (e)) at higher energies $(25-50 \mathrm{keV})$, there are double footpoint sources and a Masuda-type (Masuda et al. 1994; Nitta et al. 2010, e.g.,) coronal source located $20^{\prime \prime}=15 \mathrm{Mm}$ above the SXR (6-10 keV) loop-top. This separation is twice that of the Masuda case.

The earlier C4.5 flare (panel (a)), though more compact, exhibits surprisingly similar emission, including the additional X-ray source within the flux rope and the Masuda-type hard X-ray (HXR) source. This suggests that the end state of the first, confined flare of a failed eruption serves as the initial state of the second, eruptive flare, making them homologous flares. Note that the weaker, southern footpoint emission is (partially) occulted by the limb, especially for the first flare.

\subsection{X-ray and EUV Height-time History}

To track various moving features, we placed eight cuts of $10^{\prime \prime}$ wide centered on the limb, as shown in Figure 2 (f).
Cut 0 is positioned along the presumable current sheet between the initial flare cusp and erupting flux rope, and is used as the fiducial line for obtaining projected heights up to 08:00 UT. Subsequent cuts are evenly spaced by $3^{\circ}$ to capture the cusp at different stages as it gradual turns toward the south. For each cut, we averaged pixels of an AIA image sequence within it in the perpendicular direction to obtain a space-time plot.

Figure 4(c) shows, for example, a base-ratio (i.e., normalized by the initial intensity profile) space-time plot from Cut 0 at $131 \AA$. It shows the eruption of the flux rope and the three-stage development (upward, downward, and upward again) of the underlying flare cusp. The peak emission at each time, marked by the small purple symbols, evidently exhibits this motion. Spacetime plots of other AIA channels are shown in Figure 13 and described in Appendix A.

Figure 4(e) shows the height-time history of the looptop emission centroids in four RHESSI X-ray bands from 3 to $25 \mathrm{keV}$ and in all AIA EUV channels. (From now on, we refer to both X-ray centroids and EUV peaks as centroids.) We find that all loop-top centroids follow the same three-stage motions. The AIA 131 and $94 \AA$ channels best exhibit this trend continuously, while in cooler channels, especially 211 and $171 \AA$, the initial ascent and subsequent descent become obscure. Note that the 3-6 keV centroid (red diamonds) cannot be identified since the impulsive phase because the RHESSI thin attenuator moves in and raises the energy threshold to 6 $\mathrm{keV}$.

We also notice a clear energy dispersion that the looptop emissions at higher temperatures or photon energies are located at greater heights, with all X-ray centroids situated above EUV centroids. For example, at its greatest height prior to the impulsive phase, the $16-25 \mathrm{keV}$ centroid is $35^{\prime \prime}=26 \mathrm{Mm}$ above its $335 \AA$ counterpart. This energy dispersion is consistent with previous observations (e.g., Veronig et al. 2006) and in line with the expected picture that higher loops are newly energized and thus are hotter and/or host nonthermal electrons of harder spectra, while lower loops are previously energized and have undergone cooling. We suggest that the socalled above-the-loop-top Masuda-type sources, including the $25-50 \mathrm{keV}$ coronal source in Figure 3(d), could be extreme cases of this general trend.

In Table 2, we list the initial heights of X-ray and EUV loop-top centroids around 04:35 UT, together with the maximum and minimum heights during their descents and the percentage height reductions. In general, the above noted energy dispersion persists and the 10-20\% descents are in agreement with earlier reports (Veronig et al. 2006). An exception is the 193 and $304 \AA$ channels because of their response to both hot and cool emissions (see Appendix A). There is a weak trend that higher energy X-ray descents start (at the maximum heights) later, but still within 5 minutes during 05:0205:07 UT, and X-ray descents end (at the minimum heights) earlier than EUV descents by up to 10 minutes.

We measured the average velocities of the loop-top centroids by fitting a piecewise linear function to the heighttime data during their initial ascent, subsequent descent, and second ascent stages. As listed on the right hand side of Table 2, the result shows a general trend of higher ve- 

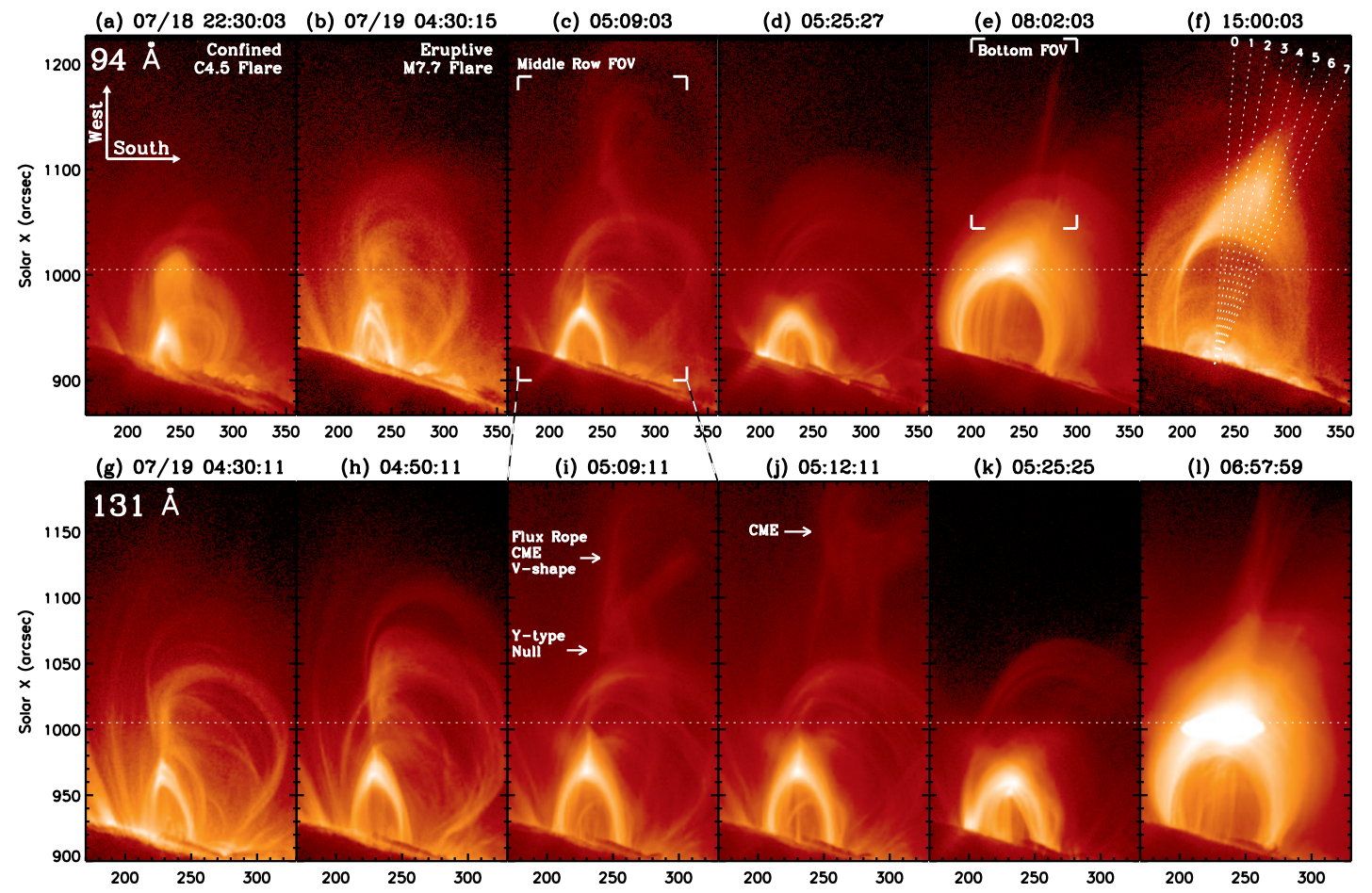

(i) 05:09:11

(j) 05:12:11

(k) 05:25:25

(1) 06:57:59

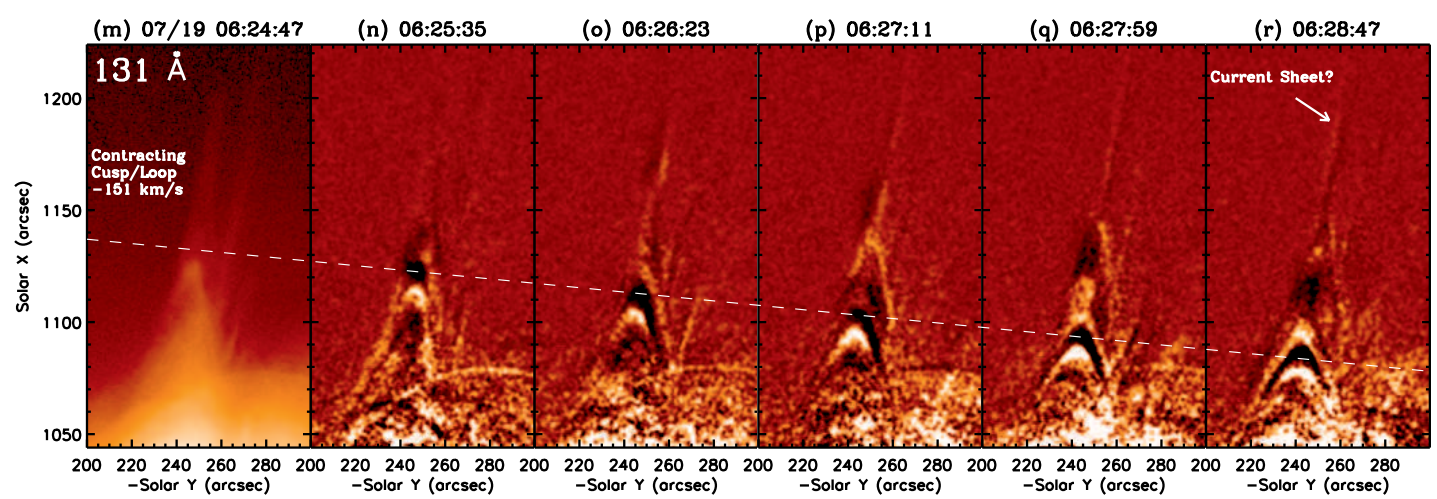

FIG. 2. - AIA images of the event, rotated to the solar west up. Top: $94 \AA$ image sequence of the earlier C4.5 flare and the M7.7 flare under study (see the associated online Movie A for composite 94 (red) and $335 \AA$ (green) images). The brackets in (c) and (e) mark the enlarged fields of view (FOV) of the middle and bottom panels, respectively. The numbered dotted lines in (f) mark the cuts for obtaining space-time plots presented in this paper. Middle: Detailed $131 \AA$ image sequence showing the upward growth of the flare cusp, followed by its rapid shrinkage around the early impulsive phase. The horizontal dotted line marks the highest position of the early cusp. Bottom: $131 \AA$ image sequence showing an example of pointed cusps contracting from a ray-like structure, presumably a reconnecting current sheet (see the associated Movie B). The first panel is an original image and the rest are running difference images. The slanted dashed line indicates an average contraction velocity of $-151 \mathrm{~km} \mathrm{~s}^{-1}$.

locities at higher energies. The X-ray descents of -11 to $-15 \mathrm{~km} \mathrm{~s}^{-1}$ are about twice faster than the EUV descents except for $193 \AA$ because of its dual temperature response. There is also a gradual decrease in velocity with time during the second ascent stage through the decay phase. These trends generally agree with previous observations (Liu et al. 2004; Veronig et al. 2006).

To follow the temporal variations of the loop-top velocities, we took time derivatives of spline fits to the centroid heights of selected channels. The resulting velocities vs. time are shown in Figure 4(c) for the 6-10 \& 10-16 keV and $131 \& 94 \AA$ channels. The outstanding feature is that the maximum descent velocities (also tabulated in Table 2) in the range of -7 to $-23 \mathrm{kms}^{-1}$ are all attained during 05:15-05:20 UT, near the onset of the impulsive phase at 05:16 UT marked by a ver- tical dashed line. This time is delayed from the initial descent by about 10 minutes. This indicates that the loop-top descent velocity, rather than its height, is more intimately correlated with the energy release rate.

We also show in Figure 4(e) the centroid heights of the upper coronal X-ray source located in the lower portion of the flux rope. A parabolic fit indicates a final velocity of $19 \mathrm{~km} \mathrm{~s}^{-1}$ at 04:50 UT, comparable to or slower than those in other events (Liu et al. 2008, 2009c; Sui \& Holman 2003). It is also $50 \%$ slower than the middle portion of the then accelerating flux rope, as indicated by the parabolic fit in Figure 13(e), which reaches $153 \pm 3 \mathrm{~km} \mathrm{~s}^{-1}$ later at 05:07 UT near the edge of AIA's FOV.

The conjugate footpoints at $25-50 \mathrm{keV}$ during the impulsive phase move away from each other at an average 


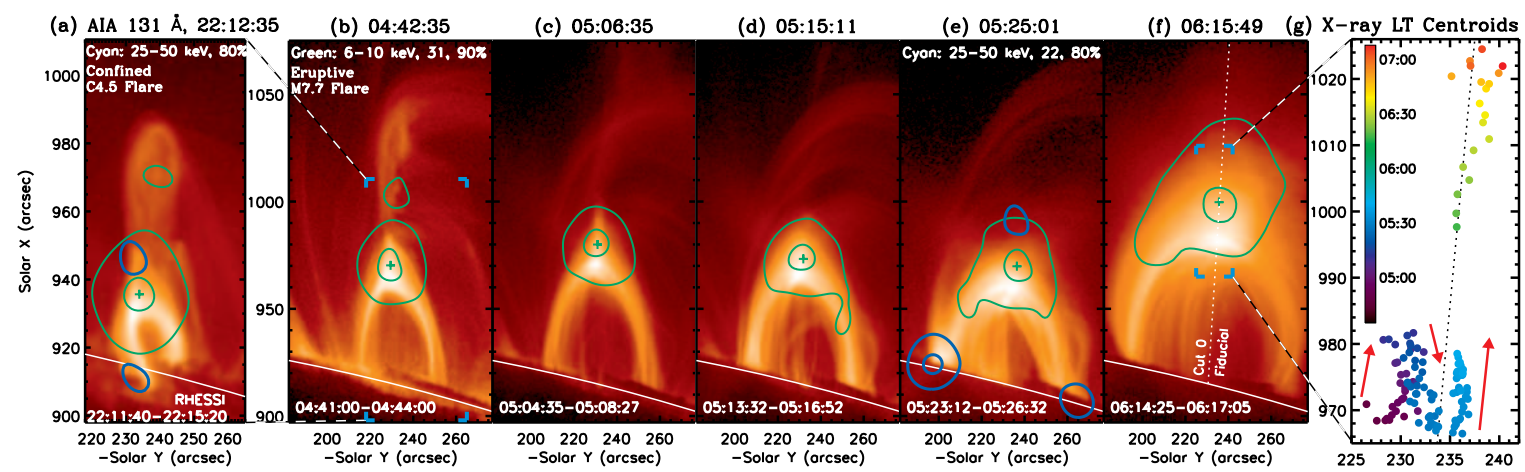

Fig. 3.- (a)-(f) Evolution of RHESSI 6-10 keV sources (green contours at 31\% and $90 \%$ of the maximum) overlaid on concurrent AIA $131 \AA$ images (see the accompanying Movie). The blue contours show $25-50 \mathrm{keV}$ emission, for both the C4.5 and M7.7 flares, at the footpoint(s) and the Masuda-type coronal source 20" above the SXR and EUV loops. (g) Color-coded temporal migration of the 6-10 keV loop-top centroid as marked by the plus sign on the left. The red arrows indicate an initial ascent followed by a descent and then another ascent. The dotted line marks the fiducial line (Cut 0) for obtaining projected heights shown in Figure 4 The first and last panels are enlarged from the bracketed regions shown in their adjacent panels.

TABLE 2

Projected Heights and Velocities of RHESSI and AiA Loop-top (LT) Centroids

\begin{tabular}{|c|c|c|c|c|c|c|c|c|c|c|}
\hline \multirow[t]{3}{*}{ Channels } & \multicolumn{4}{|c|}{ Height $(\operatorname{arcsec}=0.737 \mathrm{Mm}) /$ Time $(\mathrm{UT})$} & \multicolumn{6}{|c|}{ Velocity $\left(\mathrm{km} \mathrm{s}^{-1}\right) /$ Time $(\mathrm{UT})$} \\
\hline & \multirow{2}{*}{$\begin{array}{r}\text { Initial } \\
(\sim 04: 35) \\
\end{array}$} & \multirow{2}{*}{ Max } & \multirow{2}{*}{ Min } & \multirow{2}{*}{ Descent \% } & \multirow{2}{*}{ Initial Ascent } & \multicolumn{2}{|c|}{ Descent } & \multicolumn{3}{|c|}{ 2nd Ascent } \\
\hline & & & & & & Mean & $\operatorname{Max}$ & $-6: 00$ & $6: 00-7: 00$ & $7: 00-8: 00$ \\
\hline RHESSI & & & & & & & & & & \\
\hline $16-25 \mathrm{keV}$ & $59^{\prime \prime}$ & $77^{\prime \prime} / 05: 07$ & $60^{\prime \prime} / 05: 24$ & $22 \%$ & 8.9 & -12 & $-23 / 05: 20$ & 14 & 12 & 1.5 \\
\hline $10-16 \mathrm{keV}$ & $55^{\prime \prime}$ & $69^{\prime \prime} / 05: 06$ & $54^{\prime \prime} / 05: 21$ & $22 \%$ & 7.1 & -15 & $-19 / 05: 15$ & 14 & 10 & 3.6 \\
\hline $6-10 \mathrm{keV}$ & $53^{\prime \prime}$ & $66^{\prime \prime} / 05: 05$ & $52^{\prime \prime} / 05: 22$ & $21 \%$ & 6.0 & -11 & $-15 / 05: 18$ & 13 & 9.6 & 2.1 \\
\hline $3-6 \mathrm{keV}$ & $52^{\prime \prime}$ & $60^{\prime \prime} / 05: 04$ & & $\ldots$ & 4.1 & & $\ldots$ & & 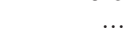 & \\
\hline AIA & & & & & & & & & & \\
\hline $193 \AA$ & & $61^{\prime \prime} / 05: 03$ & $35^{\prime \prime} / 05: 31$ & $(43 \%)$ & $\ldots$ & -13 & $-20 / 05: 19$ & .. & 4.9 & 3.0 \\
\hline $131 \AA$ & $47^{\prime \prime}$ & $56^{\prime \prime} / 05: 02$ & $43^{\prime \prime} / 05: 30$ & $23 \%$ & 4.3 & -5.9 & $-8.3 / 05: 18$ & 11 & 3.6 & 2.6 \\
\hline $94 \AA$ & $43^{\prime \prime}$ & $49^{\prime \prime} / 05: 06$ & $41^{\prime \prime} / 05: 29$ & $16 \%$ & 2.5 & -4.7 & $-7.1 / 05: 17$ & 5.9 & 3.2 & 2.6 \\
\hline $304 \AA$ & $\ldots$ & $48^{\prime \prime} / 05: 04$ & $29^{\prime \prime} / 05: 27$ & $(40 \%)$ & $\ldots$ & -5.1 & $\ldots$ & 4.6 & 4.1 & 2.4 \\
\hline $335 \AA$ & $\ldots$ & $41^{\prime \prime} / 05: 07$ & $36^{\prime \prime} / 05: 29$ & $12 \%$ & $\ldots$ & -3.9 & $\ldots$ & 4.1 & 3.5 & 3.2 \\
\hline $211 \AA$ & $\ldots$ & $\ldots$ & $31^{\prime \prime} / 05: 23$ & $\ldots$ & $\ldots$ & -6.9 & $\ldots$ & 5.0 & 4.3 & 3.2 \\
\hline $171 \AA$ & $\ldots$ & $\ldots$ & $31^{\prime \prime} / 05: 24$ & $\ldots$ & $\ldots$ & -6.0 & $\ldots$ & 5.6 & 5.0 & 3.5 \\
\hline
\end{tabular}

Note. - Centroid height uncertainties are $\lesssim 2^{\prime \prime}$ for RHESSI and $\lesssim 1^{\prime \prime}$ for AIA. Velocity uncertainties are on the order of $10 \%-15 \%$.

velocity of $13 \mathrm{kms}^{-1}$ (see Figure $4(\mathrm{e})$ ), almost identical to the velocity of the simultaneous loop-top ascent (see Table 21). This is consistent with previous observations (Liu et al. 2004) and the classical picture of eruptive flares during the arcade growth phase.

\section{BI-DIRECTIONAL PLASMA OUTFLOWS}

We now turn our attention to the bi-directional plasma outflows observed by AIA. As shown in Figure 5] and its accompanying Movie, emission features move both upward and downward from above the cusp-shaped flare loops. The upward outflows, observed only when the flux rope rises toward its eruption, are mainly bright blobs (interpreted as plasmoids, top row), while the downward outflows are primarily in the form of retracting cusp-shaped loops (bottom row) observed throughout the event well into the decay phase. Among the downward retracting loops, those seen at low altitudes tend to have lower speeds $\left(\lesssim 60 \mathrm{~km} \mathrm{~s}^{-1}\right)$ and gradually decelerate when approaching and piling up onto the apex of the flare arcade, while those originating from high altitudes tend to have higher speeds ( $\left.\gtrsim 100 \mathrm{~km} \mathrm{~s}^{-1}\right)$ and decelerate more rapidly. According to these observational distinctions, we call the former slow loop shrinkages and the latter fast loop contractions, although they might share a common physical origin as discussed in Section 6. The former are likely analogous to shrinkages observed at SXR and other wavelengths (Svestka et al. 1987), while the latter, especially those occurring after the flare arcade has formed, are likely so-called supra-arcade downflow loops (Savage \& McKenzie 2011).

To track these moving features, we obtained space-time plots from the cuts defined in Figure2(f) using the $131 \AA$ channel because it provides the best coverage of these features. We then applied running ratio, namely, dividing the intensity profile at each time by its previous neighbor, which highlights moving features as intensity tracks (see, e.g., Figure [4(d)). We exhaustively identified such tracks of more than $10^{\prime \prime}$ long corresponding to unique moving features. If one feature is captured by multiple cuts, we included only the most complete track. Following Warren et al. (2011), we fitted the projected height $h$ of each track by a function of time $t$,

$$
h(t)=h_{0}+v_{T} t+a_{0} \tau^{2}\left(e^{-t / \tau}-1\right),
$$

which gives the instantaneous velocity

$$
v(t)=v_{T}-a_{0} \tau e^{-t / \tau},
$$

and acceleration

$$
a(t)=a_{0} e^{-t / \tau}
$$

where $h_{0}$ and $a_{0}$ are the initial height and acceleration, $v_{T}$ is the terminal velocity, and $\tau$ is the $e$-folding decay 

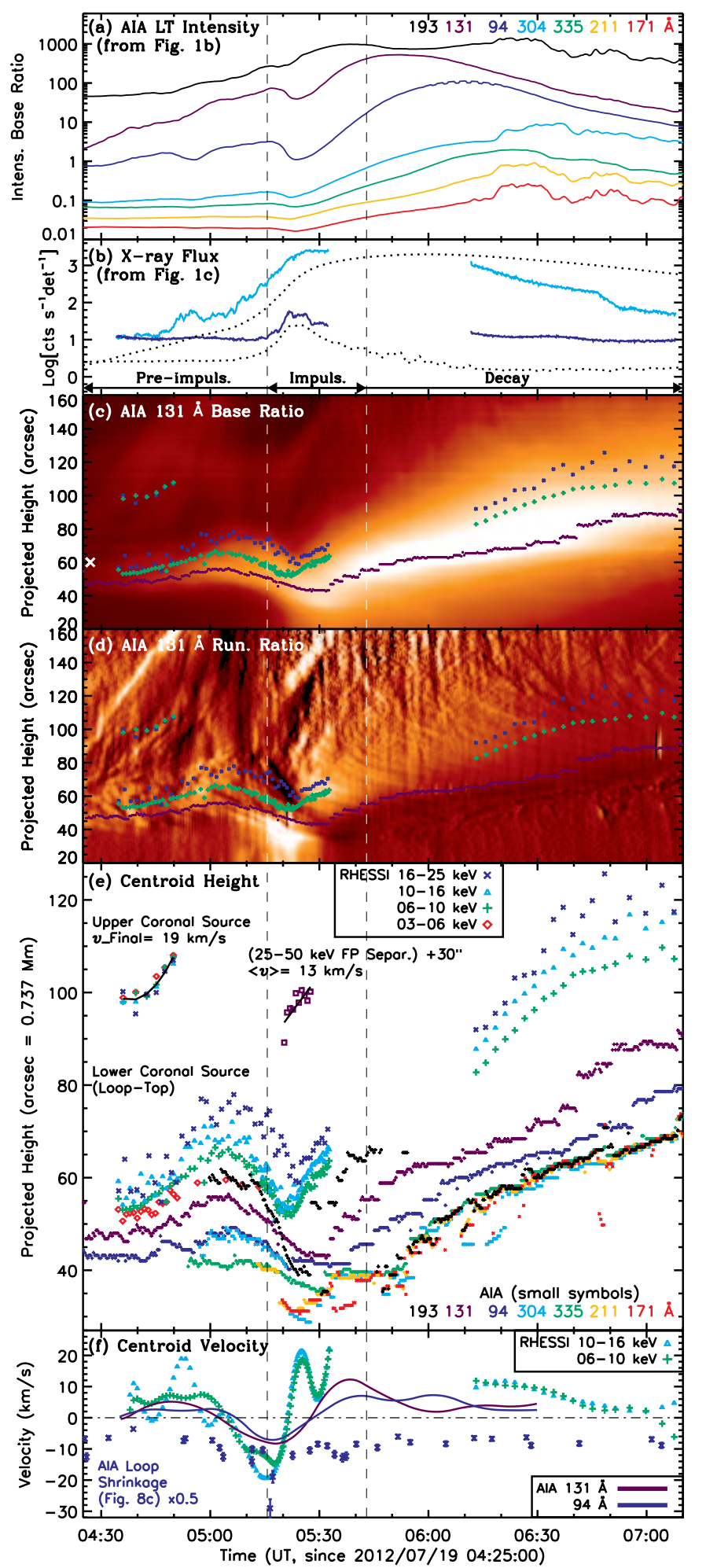

Fig. 4.- X-ray and EUV source motions. (a) and (b) Subsets of light curves from Figures 1 (b) and (c). (c) and (d) Base and running ratio space-time plots of the AIA $131 \AA$ channel from Cut 0 , overlaid with selected centroid positions from (e). (e) Projected heights of the emission centroids of the upper and lower coronal sources from RHESSI (large symbols, greater heights) and of the loop-top peaks from AIA (small symbols, lower heights) Also shown is the $25-50 \mathrm{keV}$ footpoint separation (purple squares) shifted upward by $30^{\prime \prime}$. (f) Velocity of centroids from spline fits to selected channels in (e). The blue symbols are the initial velocities of individual but rescaled by a factor of 0.5 . $\begin{array}{lllll}\text { (a) } 04: 33: 11 & \text { (b) } 04: 33: 23 & \text { (c) } 04: 33: 47 & \text { (d) } 04: 33: 59\end{array}$

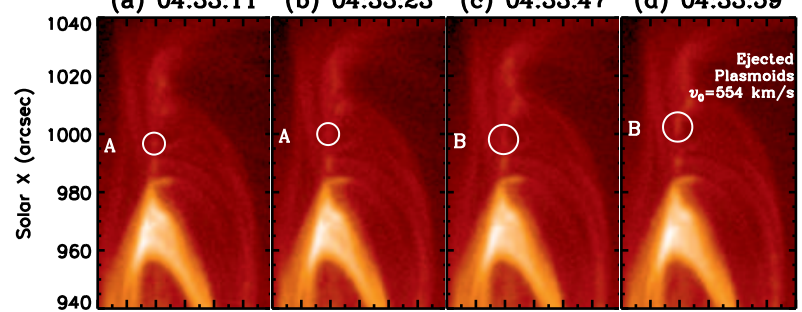

$\begin{array}{lllll}\text { (e) } 04: 51: 35 & \text { (f) } 04: 51: 47 & \text { (g) } 04: 51: 59 & \text { (h) 04:52:11 }\end{array}$
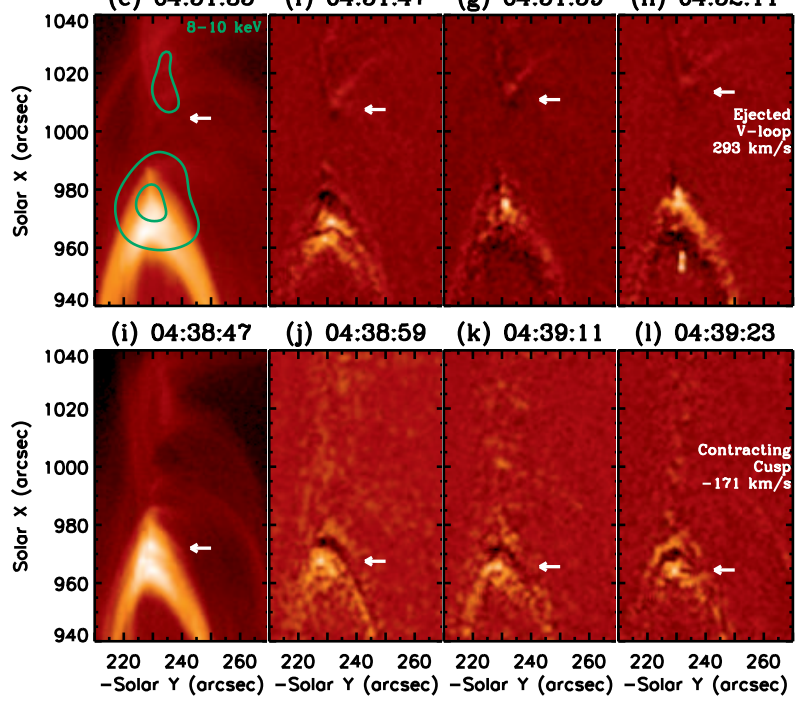

FIG. 5.- AIA $131 \AA$ images (see the accompanying Movie) showing upward moving blobs (top, labeled A and B) and V-shapes (middle) and downward contracting cusps (bottom). Their spacetime fits are shown in Figure 7 (a). The green contours show two distinct 8-10 keV RHESSI sources from Figure 10 (c). The first row and column are original images and the rest are running difference images.

time that characterizes the observed decreasing acceleration (or deceleration) with time. We present below the kinematics of the features categorized above and focus on their initial velocities $v_{0}=v_{T}-a_{0} \tau$ and accelerations $a_{0}$.

\subsection{Slow Downward Loop Shrinkages}

Figure 6] shows kinematic measurements of the slow loop shrinkages. These loops traverse a hot (up to $T \sim 30 \mathrm{MK}$; see Figure 11) loop-top region, which is bright only in the hottest channels of 193, 131, and $94 \AA$, and descend toward the apex of the flare arcade seen in cooler channels, where they fade below detection (see Figure 13). They generally start at a low velocity with a median of $-17 \mathrm{~km} \mathrm{~s}^{-1}$ (+/ - for upward/downward) and decelerate to typically $-5 \mathrm{~km} \mathrm{~s}^{-1}$ within $0.5-2.5 \mathrm{~h}$. Their median initial deceleration is $0.014 \mathrm{~km} \mathrm{~s}^{-2}$.

Such shrinkages are persistent throughout the flare, not only during the decay phase when the overall loop-top emission develops upward as previously observed (Forbes \& Acton 1996), but also during the preimpulsive and impulsive phases when the loop-top undergoes its initial ascent and subsequent descent. 50 shrinkages are identified during 04:00-16:00 UT with an average occurrence rate of once per 7.7 minutes up to 10:00 UT but more frequent around the impulsive phase (Figure 6(b)).

Regardless of the direction of motion of the loop-top 

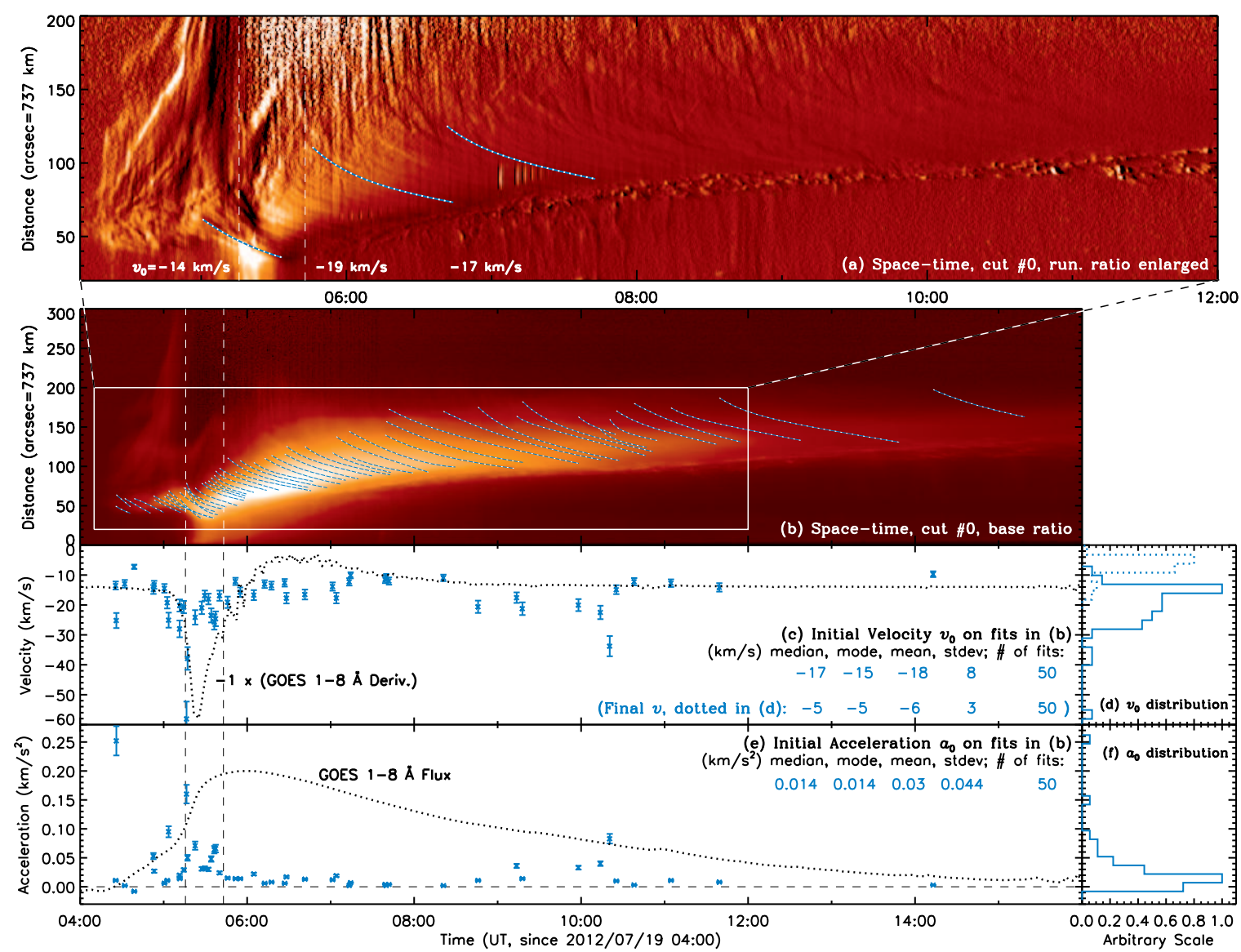

FIG. 6. - Kinematic measurements of slow loop shrinkages. (a) Running ratio space-time plot enlarged for the boxed region in (b), overlaid with examples of fits to shrinkage tracks labeled with their initial velocities. (b) Base ratio 131 A space-time plot from Cut 0 overlaid with all 50 fits performed. (c) Initial velocities $v_{0}$ of the fitted tracks in (b) as a function of time. (d) Histograms of $v_{0}$ (solid) and the final velocity $v_{f}$ (dotted) measured at the end of each track, with their statistical properties (median, mode, mean, standard deviation, and total number of fits) listed in (c). (e) and (f) same as (c) and (d), but for the initial acceleration $a_{0}$. The black dotted lines in (c) and (e) are the time derivative (inverted) and original GOES 1-8 $\AA$ flux. The two vertical dashed lines mark the impulsive phase.

emission, the initial height of loop shrinkage generally increases with time. The initial velocity $v_{0}$ exhibits more variability, as shown in Figure 6(c), and appears to be positively correlated with the flare energy release rate indicated by the time derivative of the GOES flux (black dotted line). In particular, near the onset of the impulsive phase at 05:16 UT (vertical dashed line), $v_{0}$ increases rapidly and reaches a maximum of $-58 \mathrm{~km} \mathrm{~s}^{-1}$. There is a similar temporal correlation with the initial deceleration $a_{0}$ (panel (d)) that also generally correlates with $v_{0}$.

\subsection{Fast Downward Loop Contractions}

Figure 7 shows, in the same form as Figure 6, kinematic measurements of fast downward loop contractions as well as upward plasmoid ejections that will be examined in Section 4.3. We color-code fits to tracks of different categories: upward ejections in red and downward contractions in cooler colors. For the latter, we use green for early contractions that occur simultaneously with upward ejections and originate from lower heights $h<100^{\prime \prime}$, and blue for later contractions that occur without observed upward counterparts (possibly out of AIA's FOV, after the flux rope has erupted) and originate from greater heights $h>100^{\prime \prime}$. We identified 29 green tracks and 174 blue tracks during 04:00-16:00 UT.
As shown in Figure 7(c), the fast downward contractions, especially those later blue-colored ones, start well above the hot loop-top region and travel into it with deceleration for some distance before fading below detection. Their final heights are generally above the original heights of slow loop shrinkages (see Figure 6(b)).

In original AIA images, the fast contracting features are usually bright, cusp-shaped loops (see Figure 2, bottom), but occasionally dark, tadpole-like voids (McKenzie \& Hudson 1999; Innes et al. 2003; Cassak \& Drake 2013). In running ratio space-time plots (Figure 7, top), the former each produce a bright track followed by a recovering dark track, while the latter produce tracks of reversed order. We treat them all as contracting loops and do not distinguish their differences that are beyond our scope.

As shown in Figure 7(c), the early, green-colored contractions have short ranges of $10^{\prime \prime}-20^{\prime \prime}$, because of the compactness of the flare at this time, while the later, blue-colored contractions travel great distances up to $200^{\prime \prime}=150 \mathrm{Mm}$. However, their statistical medians, modes, and means of the initial velocities are quite similar within 200-300 $\mathrm{km} \mathrm{s}^{-1}$ (Figures 7(d) and (e); Table 3), suggestive of a common origin of these contractions possibly as reconnection outflows. The initial decelerations of the early contractions are, on the other hand, 

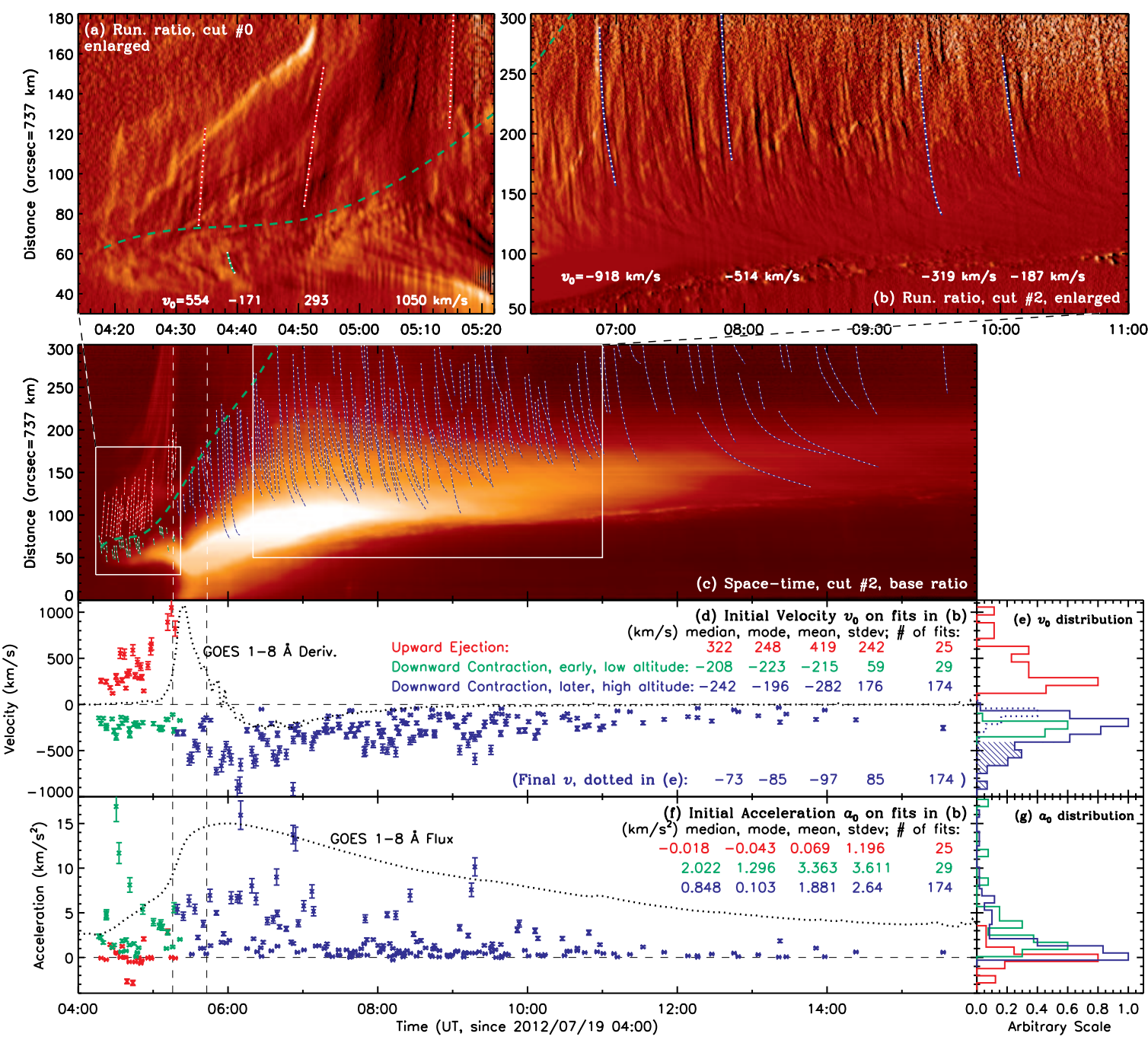

FIG. 7.- Same as Figure 6 but for fast upward plasmoid ejections and downward loop contractions. (a) and (b) Running ratio spacetime plots obtained from Cuts 0 and 2, enlarged for the two boxed regions in (c). Overlaid with examples of color-coded fits, (a) shows simultaneous upward ejections (red dotted) and downward contractions of early, low-lying loops (green dotted), and (b) shows downward contractions of later, high-lying loops (blue dotted) from well above the flare arcade. Example images corresponding to the fits in (a) and (b) are shown in Figures 5 and 2 (bottom), respectively. (c) Base ratio $131 \AA$ space-time plot from Cut 2 overlaid with all fits to these identified features. The thick green dashed line is a spline fit to the initial heights of bi-directional outflows, as an estimate to the position of reconnection. (d) $-(\mathrm{g})$ Initial velocities $v_{0}$ and accelerations $a_{0}$ of the color-coded fits in (c) as a function of time, together with their histograms on the right. The blue dotted line in (e) is for the final velocities of the late, high-lying loop contractions. The short, vertical stripes in (a) with a periodicity of $\sim 1$ minute between 04:45-05:15 UT are possibly due to quasi-periodic flare pulsations (Nakariakov \& Melnikov 2009), not to be confused with the tracks of steep slopes of plasma outflows.

TABLE 3

Kinematic Statistics of Bi-directional Outflows

\begin{tabular}{|c|c|c|c|c|c|c|c|c|c|c|c|c|c|c|c|c|}
\hline \multirow{2}{*}{$\begin{array}{l}\text { Features } \\
\text { (\# of Fits) }\end{array}$} & \multirow{2}{*}{$\begin{array}{c}\text { Interval }(a) \\
\text { (minutes) }\end{array}$} & \multicolumn{5}{|c|}{ Initial Velocity $\left(\mathrm{km} \mathrm{s}^{-1}\right)$} & \multicolumn{5}{|c|}{ Final Velocity $\left(\mathrm{km} \mathrm{s}^{-1}\right)$} & \multicolumn{5}{|c|}{ Initial Acceleration $\left(\mathrm{km} \mathrm{s}^{-2}\right)$} \\
\hline & & Med. & Mod. & $\sigma$ & Max/Time & Min & Med. & Mod. & $\sigma$ & Max/Time & Min & Med. & Mod. & $\sigma$ & Max/Time & $\pi$ \\
\hline Fiecte o Pla & 2.4 & 322 & 248 & 242 & & 121 & 343 & 320 & 250 & & 70 & -0.018 & -0.043 & 1.2 & & \\
\hline & 7 & -17 & -15 & 8 & & -7 & -5 & -5 & 3 & & -2 & 0.0 & 0.014 & 044 & & \\
\hline & & & & & & & & & & & & & & & & \\
\hline & & 208 & -231 & 59 & & & -89 & & 58 & & -26 & & & & & \\
\hline $1 F$ & 2.0 & -242 & -158 & 176 & $6: 52$ & -34 & -73 & -113 & 85 & $5: 32$ & -1 & 0.8 & 0.103 & 2.64 & $15.9 / 06: 10$ & -0.297 \\
\hline
\end{tabular}

Note. - Abbreviations: Med. for Median, Mod. for Mode. (a) Average occurrence interval up to 10:00 UT. 
about twice higher than those of the later ones, with a median of 2.022 vs. $0.848 \mathrm{~km} \mathrm{~s}^{-2}$ (Figures 7 (f) and (g)).

The later, high-altitude contractions have a mode velocity $-158 \mathrm{~km} \mathrm{~s}^{-1}$ that is similar to the median velocity $-150 \mathrm{~km} \mathrm{~s}^{-1}$ of supra-arcade downflows (Savage \& McKenzie 2011; Warren et al. 2011). However, their median and mean velocities, -242 and $-282 \mathrm{~km} \mathrm{~s}^{-1}$, are nearly twice greater. This is due to the high velocity tail with 36 out of 174 or $21 \%$ contractions being faster than $-400 \mathrm{~km} \mathrm{~s}^{-1}$ (hatched area in Figure 7(e)), reaching a maximum of $-918 \mathrm{~km} \mathrm{~s}^{-1}$. We ascribe this difference to AIA's improved capabilities that allow us to detect such contractions in their early stages when their velocities are high and emissions are weak.

The initial velocities and decelerations of the earlier and later fast contractions, when taken together, generally increase with time up to $\sim 06: 10$ UT and then decrease. This maximum time is delayed by almost $1 \mathrm{~h}$ from that of the slow loop shrinkages and the onset of the HXR burst represented by the GOES flux derivative shown in Figure 6(c). The temporal variations here seem to be more closely correlated with the GOES flux itself. The late-phase decreases of the initial velocities and decelerations are physically reasonable as energy release subsides, but there is an observational bias for those tracks starting at the edge of AIA's FOV which may have decelerated before they are first detected.

Figure 8 shows the distributions of fractional height reductions of the downward loop shrinkages and contractions, defined as $\left(h_{0}-h_{f}\right) / h_{0}$, where $h_{0}$ and $h_{f}$ are the initial and final projected heights. The slow shrinkages (light blue) and later, high-altitude fast contractions (blue) have similar distributions with medians of 0.3 and 0.26 , respectively, which are comparable to those observed in SXRs (Forbes \& Acton 1996; Reeves et al. 2008) and predicted theoretically (Lin 2004). The early, low-altitude fast contractions (green) have a median of twice smaller, but comparable to the 10-20\% descent of the overall loop-top emission (Table 21). Meanwhile, the later fast contractions have a sizable fraction of $16 \%$ (27 out of 174) with height reductions more than 0.4 (up to 0.7 ), which is larger than predicted. Note that these observed values are lower limits, because loops, especially those detected at the edge of AIA's FOV, could continue contraction before and after their observed intervals when their emission remains below detection.

\subsection{Fast Upward Plasmoid Ejections}

As shown in Figure 7 upward plasmoid ejections appear as steep, elongated tracks (red fits). Compared with the downward loop contractions (green fits) at the same time, they travel greater distances $\left(20^{\prime \prime}-\right.$ $80^{\prime \prime}$ vs. $10^{\prime \prime}-20^{\prime \prime}$ ), with larger initial velocities (median: 322 vs. $-208 \mathrm{~km} \mathrm{~s}^{-1}$ ) but smaller decelerations (median: -0.018 vs. $2.022 \mathrm{~km} \mathrm{~s}^{-2}$ ). (The opposite signs of the acceleration and velocity here indicate deceleration.) Some upward ejections even experience acceleration. Such differences, especially the ratio of their median velocities of $208 / 322=2 / 3$, are consistent with previous observations and simulations of oppositely directed reconnection outflows (Takasao et al. 2012; Bárta et al. 2008; Shen et al. 2011; Murphy et al. 2012; Karpen et al. 2012). These

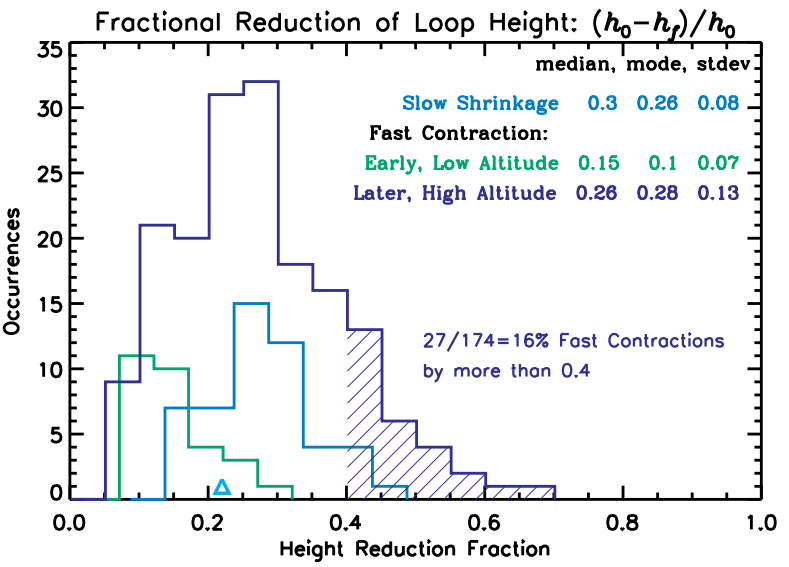

Fig. 8.- Histograms of fractional height reductions of slow shrinkages (light blue) and early, low-altitude (green) and later, high-altitude (blue) fast contractions. The hatched area indicates $16 \%$ of the later fast contractions with reductions greater than 0.4 . The cyan triangle marks the descent fraction of the $10-16 \mathrm{keV}$ loop-top from Table 2

differences are likely due to different environments: the upflow runs into a region of (partially) open field lines with low plasma density, while the downflow impinges on high density, closed flare loops that can cause strong deceleration.

The starting heights of these upward ejections and their downward counterparts (Figure $7(\mathrm{c})$ ) both increase with time. This suggests an upward development of the reconnection site situated between the opposite outflows (e.g., Shen et al. 2011). We estimated the height of the reconnection site from a spline fit, shown as the green dashed line in Figures 7 (a)-(c), to the initial heights of the bi-directional fast outflows. Its initial velocity of a few $\mathrm{km} \mathrm{s}^{-1}$ is similar to that of the initial loop-top ascent, suggesting the upward development of reconnection being its underlying mechanism. This estimated reconnection site leaves the AIA FOV at about 06:40 UT.

During 04:15-05:25 UT, we identified 25 upward ejections (red) and 29 downward, early fast contractions (green), at average occurrence rates of once every 2.4 and 2.2 minutes, respectively. These rates are comparable to that of the later fast contractions (blue, up to 10:00 UT) of once every 2.0 minutes and to those in MHD simulations (Bárta et al. 2008) 4 The persistence throughout the entire flare of such contractions also agrees with the statistical result from Yohkoh (Khan et al. 2007).

The initial velocity of upward ejections, as shown in Figure 7(d), increases with time and seems to be correlated with the height of the overlying flux rope as it evolves into eruption. In particular, there is a rapid velocity increase at the onset of the impulsive phase, reaching $1050 \mathrm{~km} \mathrm{~s}^{-1}$. This aspect of this event is independently studied by R. Liu (2013).

\section{SPATIAL DISTRIBUTION OF ENERGY AND TEMPERATURE DEPENDENT EMISSION}

\subsection{RHESSI X-ray Spectra}

\footnotetext{
4 The $\sim 2$ minute intervals of fast contractions are comparable to the typical periodicities of recently detected quasi-periodic fast-mode magnetosonic wave trains (Liu et al. 2011b, 2012d; Ofman et al. 2011; Shen \& Liu 2012) that are correlated with flare pulsations. This may indicate that those waves are triggered by the energy release episodes associated with these contractions.
} 
To infer the overall properties of nonthermal particles and thermal plasma, we analyzed spatially integrated RHESSI spectra of individual detectors following the procedures detailed in Liu et al. (2008) and Milligan \& Dennis (2009).

Figure 9 shows example spectra in three phases of the flare. (1) During the pre-impulsive phase (04:59:0005:01:32 UT), we fitted the spectrum with two isothermal functions combined: a warm component (red dotted) of temperature $T=13 \mathrm{MK}$ and emission measure $\mathrm{EM}=0.37 \times 10^{48} \mathrm{~cm}^{-3}$ plus a hot component (green dotted) of $30 \mathrm{MK}$ (also called super-hot, Caspi \& Lin 2010) and a 40 times smaller EM. If we were to replace the hot component with a power-law $\propto E^{-\gamma}$, we would have a steep slope of $\gamma=7.6$. (2) During the impulsive phase (05:25-05:26 UT), we used an isothermal $(T=22 \mathrm{MK}$, $\mathrm{EM}=2.8 \times 10^{48} \mathrm{~cm}^{-3}$ ) plus power-law (blue dotted, $\gamma=3.8$ ) model. (3) During the decay phase, the data can be fitted with one isothermal function of a slightly reduced temperature of $18 \mathrm{MK}$ but twice higher EM of $5.5 \times 10^{48} \mathrm{~cm}^{-3}$. Note that at $E>25 \mathrm{keV}$, the power law in the impulsive phase is more than an order of magnitude higher than the thermal component. This indicates that the 25-50 keV Masuda-type loop-top source shown in Figure 3 is primarily nonthermal and likely a particle acceleration region itself (e.g., Liu et al. 2008; Krucker et al. 2010).

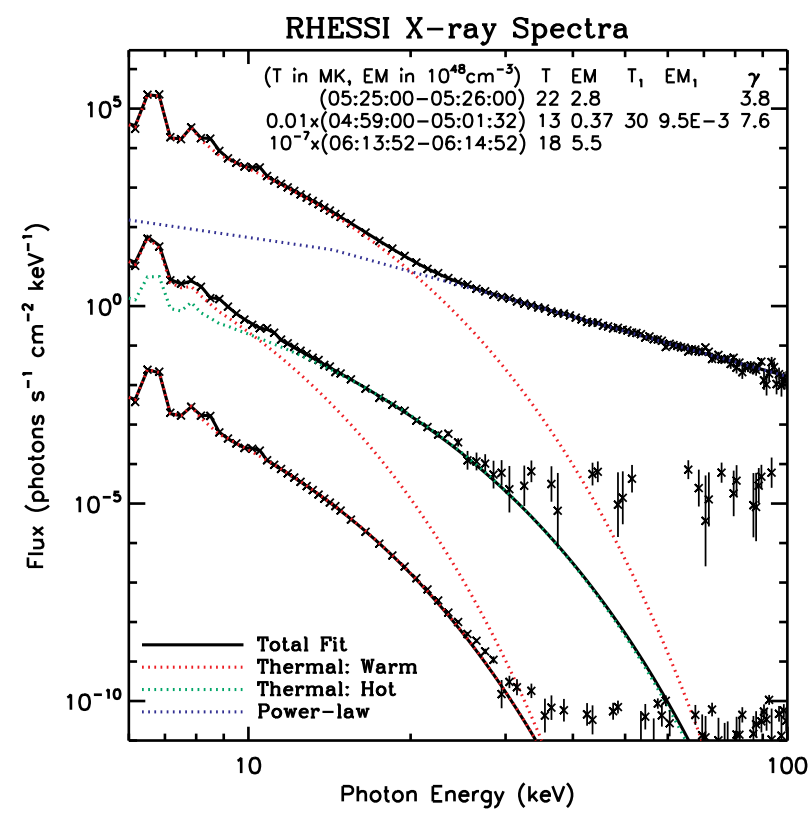

FIG. 9.- RHESSI spectra at three selected times before, during, and after the impulsive phase. The lower two spectra are vertically shifted by decades to avoid overlap. Colored dotted lines are isothermal and power-law fits whose parameters are listed on the top. The black solid lines are the total fits.

We applied such spectral fits throughout the flare and the temporal variations of the fitting parameters are shown in Figures 1(d) and (e). In general, the warm thermal component is persistent in time, and both its $T$ and EM (orange plus signs) increase through the HXR burst followed by a more gradual decrease. A similar trend is present for the hot thermal component (red crosses) during the pre-impulsive phase. A thermal fit to GOES data gives a somewhat lower $T$ but higher EM (black dotted lines), because of its well-known preferential response to relatively cooler plasma. The power-law component (blue) displays a common hardening trend before the RHESSI night data gap.

\subsection{Energy Dependence of Double Coronal Sources}

In Section 3.2 we have examined the energy-dependent height distribution of the loop-top X-ray source (lower coronal source). Here we extend this analysis to the upper coronal source during the pre-impulsive phase and compare the two sources together.

As shown in Figures 10(a) and (b), the higher-energy emission (12-15 keV, blue contours) of the lower source is located at higher altitudes than lower-energy emission (3-8 keV, green contours), while the upper source has an opposite trend. That is, the two sources are closer to each other at higher energies, as can be better seen in panel (d) from the linear fits to their centroid heights as a function of logarithmic energy. This implies higher temperatures of the thermal plasma and/or harder spectra of the nonthermal particles within the inner regions. Similar RHESSI observations have been reported (Sui \& Holman 2003; Liu et al. 2008, 2009c) and interpreted as evidence of magnetic reconnection and associated energy release being located between the double coronal sources.

At the highest energies $(\gtrsim 10 \mathrm{keV})$, this trend seems to be reversed, especially for the upper source. In previous observations (e.g., Figure 4 in Liu et al. 2008), more obvious reversals occurred at slightly higher energies of 15-20 keV and were interpreted as a transition from the low-energy thermal regime to the high-energy nonthermal regime, in which greater stopping distances of higher energy electrons can cause higher energy bremsstrahlung $\mathrm{X}$-rays being located farther away. We further suggest that this reversal may be an indication of the two Xray sources being spatially separated, instead of merging together at the highest energies; so are the locations of primary heating and particle acceleration from magnetic reconnection. This is supported by the separate temperature peaks at the two sources as shown in Figures 111(a) and $(\mathrm{h})$ and discussed in Section 5.3 .

\subsection{AIA Temperature Maps}

To infer the spatial distributions of temperature $T$ and emission measure (EM), we employed a forward-fitting algorithm using AIA filter ratios Aschwanden et al. 2011; for a different approach, see Battaglia \& Kontar 2012). This algorithm assumes a Gaussian differential emission measure that has a peak EM at temperature $T$. Such a model provides the simplest description of the EM weighted temperature distribution along the line of sight.

Figure 11 (left) shows examples of $T$ and EM maps. Early in the event at 04:49 UT, the highest temperature of $\log T=6.95$ or $T=8.9 \mathrm{MK}$ is located in the rising flux rope and the underlying flare cusp, but EM is highly concentrated in the latter. The temperature then increases with time especially in the flare cusp, reaching $\log T=7.45$ or $T=28.2 \mathrm{MK}$ at 05:09 UT, close to the $35 \mathrm{MK}$ of the hot RHESSI component at this time (see Figure 1(d), red crosses). In the decay phase, the highest temperatures are located in the outer layer of 

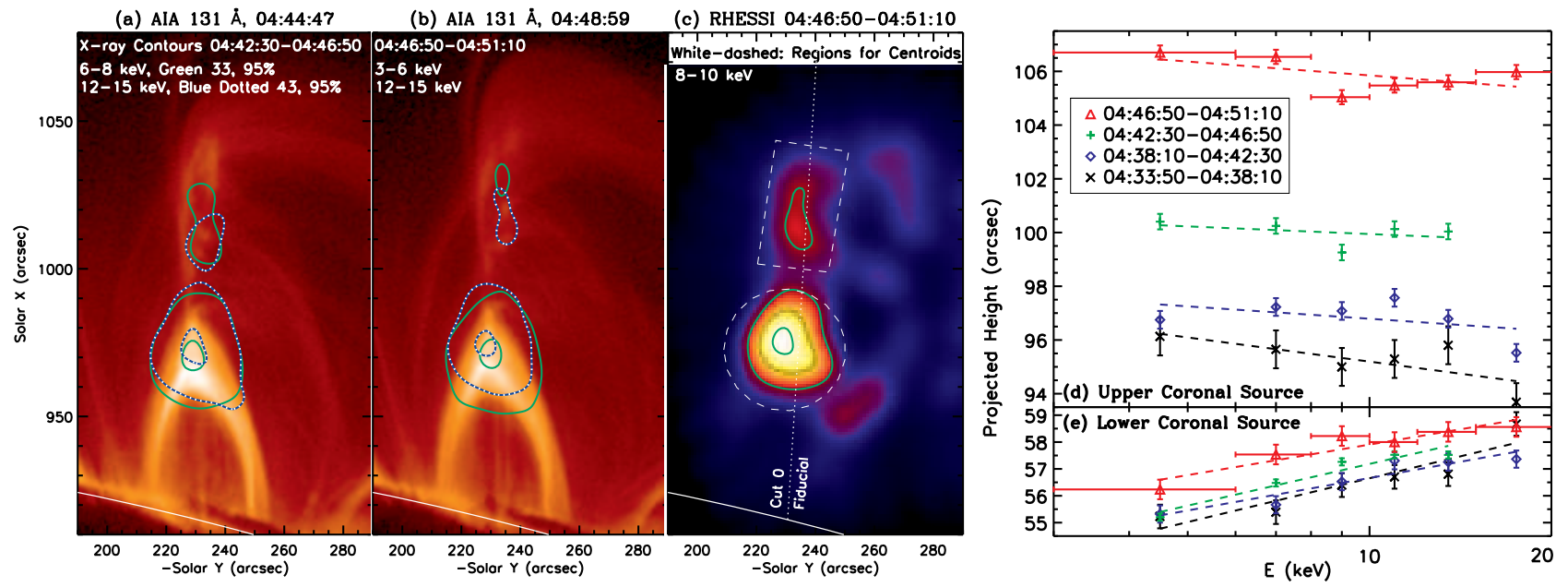

FIG. 10.- Energy dependence of the double coronal X-ray sources likely located in the oppositely directed reconnection outflows. (a) and (b) RHESSI contours overlaid on AIA 131 A images. The higher energy sources (blue dotted contours at $43 \%$ and $95 \%$ of the image maximum) are closer toward one another than the lower energy sources (green contours). (c) Contoured 6-8 keV image at the time of (b). (d) and (e) Projected heights along the fiducial Cut 0 of the centroids of the two sources enclosed by the white dashed lines in (c). The result is color-coded for four consecutive times overlaid with linear fits in dashed line. The red horizontal error bars indicate energy bins.

the flare arcade and the lower portion of the contracting cusps (ray-like features) above it. The EM in the latter is nearly four orders of magnitude lower than in the central arcade, similar to those of hot fans of rays observed by Yohkoh (Svestka et al. 1998). The considerable increase of EM and thus density in the flare loops from panel (d) to (e), prior to the impulsive phase, suggests chromospheric evaporation driven by thermal conduction (Liu et al. 2009b; Battaglia et al. 2009) or by Alfvén waves (Haerendel 2009), rather than electron beam heating that may be important later in the presence of HXR footpoints.

To better follow the history of $T$ and EM, we obtained their space-time plots, as shown in Figures 11(g) and $(\mathrm{k})$, from corresponding maps using Cut 0 . We identified the maximum temperature and EM at each time and show their projected heights as orange and black symbols in panel (p). In general, both peaks follow the same upward-downward-upward motions as the loop-top emission centroids. The temperature peaks are near the RHESSI 16-25 keV centroids (blue), while the EM peaks are close to or slightly lower than the AIA $131 \AA$ centroids (purple) at lower heights.

The peak offset of the temperature and EM can be better seen in their height distributions at selected times shown in Figures 11(h) and (l). It explains the observed energy dispersion of the X-ray loop-top centroids shown in Figure 4(e) because of the exponential shape of thermal bremsstrahlung spectra (equation (41)). As shown by the green and red lines in the middle of Figure 9 . a higher temperature but lower EM produces a harder (shallower) spectrum of lower normalization that dominates at high energies, while a lower temperature but higher EM produces a softer (steeper) spectrum of higher normalization that dominates at low energies. The temperature peak being located above the EM peak thus shifts the higher energy X-rays toward greater heights. Otherwise, if the temperature and EM peaks are cospatial, they would dominate X-rays at all energies and there would be no separation of centroids with energy. We note in Figures 11 (h) that the EM decreases more gradually near the flux rope. This may lead to the less pronounced energy dispersion of the upper coronal source there than the lower (loop-top) source (see Figure 10).

As a proof of concept, we modeled the observed Xrays of energy $E$ with thermal bremsstrahlung radiation (Tandberg-Hanssen \& Emslie 1988, p. 114):

$$
I_{\mathrm{SXR}} \propto(\mathrm{EM}) \frac{\exp (-E / k T)}{E \sqrt{T}} g(E / k T),
$$

where EM is the emission measure and $g(E / k T)=$ $(k T / E)^{2 / 5}$ is the Gaunt factor. The resulting X-ray profiles at two selected times are shown in Figures 11)(i) and $(\mathrm{m})$ for the corresponding $T$ and EM profiles. As expected, the higher energy $20 \mathrm{keV}$ emission (blue) is dominated by the temperature peak at a higher altitude, while the $3 \mathrm{keV}$ emission (red) is dominated by the EM peak at a lower altitude. Their emission peaks, marked by open circles, differ in height by $\Delta h=7^{\prime \prime}$ and $46^{\prime \prime}$ for the two times. Their peak heights are repeated in panel (p) and are close to those of observed loop-top centroids.

As shown in Figures 11(g) and (p), the high temperature region and particularly the temperature peak are close to the X-ray loop-top centroids but always below the reconnection site (green dashed line). This indicates that primary plasma heating takes place in reconnection outflows. To illustrate this, we can follow a contracting loop and the temperature variation it senses as it travels away from the reconnection site. A selected track from Figure 7(c) is shown here as the blue dotted arrow. The temperature history on its path, as shown in Figures 11(h) and (j), indicates rapid heating at an average rate of $3.2 \mathrm{MK} \mathrm{min}^{-1}$ from 3.5 to $28 \mathrm{MK}$ over 8 minutes and $\Delta h=90 \mathrm{Mm}$. This example gives us a sense of the heating rate averaged along the line of sight, not necessarily of a specific loop.

Likewise, the track of a slow loop shrinkage at lower altitudes reveals cooling at an average rate of $-0.32 \mathrm{MK} \mathrm{min}^{-1}$ from 25 to $7.9 \mathrm{MK}$ within 54 minutes. The rate is $-1 \mathrm{MK} \mathrm{min}^{-1}$ earlier during the impulsive phase in the 28-9 MK range and $-0.1 \mathrm{MK} \mathrm{min}^{-1}$ later during 07:00-08:30 UT in the 14-7 MK range. These 

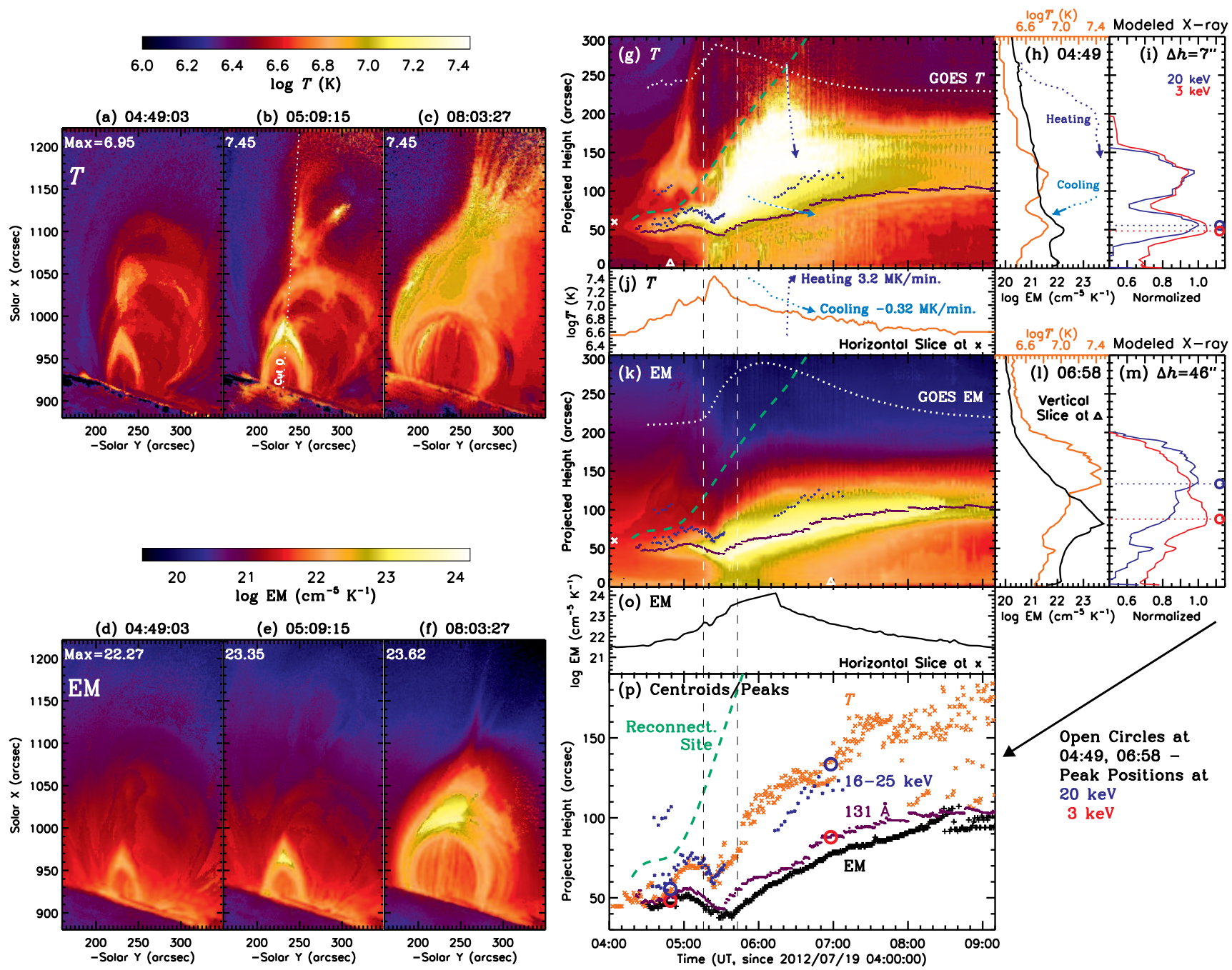

FIG. 11.- Maps of temperature $T(\mathrm{a}-\mathrm{c})$ and emission measure EM (d-f) inferred from AIA filter ratios at three selected times on logarithmic scales. (g) and (k) Space-time plots of $T$ and EM maps from Cut 0 as shown in (b). The white dotted lines are temperature and emission measure from GOES data as shown in Figures 1 d d ) and (e). The first side panels ((h) and (l)) on the right show the height distributions of $T$ (red, top scale) and EM (black, bottom scale) at the time marked by the triangle on the left. The second side panels $((\mathrm{i})$ and $(\mathrm{m})$ ) show the modeled corresponding thermal X-ray emission at two photon energies, 3 and $20 \mathrm{keV}$. The open circles mark the heights of the emission peaks that are also shown in $(\mathrm{p})$. (j) and (o) Temporal profiles $T$ and EM, obtained from a horizontal slice of the corresponding space-time plots above at height $h_{\text {ref }}=60^{\prime \prime}$ marked by the cross. The arrowed blue and light blue dotted lines in (g) are selected tracks of a fast contraction and slow shrinkage from Figures 7 and 6 The temperatures sampled by these tracks are shown as a function of time and distance in $(\mathrm{j})$ and $(\mathrm{h})$, respectively, indicating heating and cooling experienced by these loops as they travel. (p) Projected heights of the maxima of $T$ (orange) and EM (black) at each time in the corresponding space-time plots. Overlaid here and in the above space-time plots are the heights of the $16-25 \mathrm{keV}$ (blue) and $131 \AA$ (purple) loop-top centroids in small symbols from Figure 4 (e), and of the inferred reconnection site in green dashed line from Figure [7(c). The vertical dashed lines mark the impulsive phase.

cooling rates are somewhat lower than previously found at even lower temperatures (Vršnak et al. 2006).

\section{CONCLUSION}

\subsection{Summary}

We have presented detailed EUV and X-ray observations of the 2012 July 19 M7.7 flare, focusing on the signatures of magnetic reconnection and associated energy release. We summarize our findings and discuss their implications as follows.

1. The V-shaped EUV emission on the trailing edge of the flux rope CME and the underlying, inverted $\mathrm{V}$-shaped flare loops, both associated with distinct $\mathrm{X}$-ray emission, suggest two oppositely oriented Ytype null points with a vertical current sheet formed in between (Figures 2 and 5 ).
2. Originating from the inferred magnetic reconnection site within the current sheet are bi-directional outflows in the forms of plasmoids and cuspshaped loops. The upward ejections have a median initial velocity of $320 \mathrm{~km} \mathrm{~s}^{-1}$ and a maximum of $1050 \mathrm{~km} \mathrm{~s}^{-1}$, while the concurrent downward cusp contractions are $\sim 2 / 3$ slower with a median of $-210 \mathrm{kms}^{-1}$. Even faster contractions up to $-920 \mathrm{~km} \mathrm{~s}^{-1}$ occur after the flux rope eruption for another $10 \mathrm{~h}$, and a sizable fraction of $21 \%$ of them have speeds $\geq 400 \mathrm{kms}^{-1}$, twice faster than previously reported (Savage \& McKenzie 2011). Such high velocities are comparable to expected coronal Alfvén speeds of $\sim 1000 \mathrm{~km} \mathrm{~s}^{-1}$ and sound speeds of $520-910 \mathrm{kms}^{-1}$ for a $10-30 \mathrm{MK}$ flaring plasma. At lower altitudes, flare loops persistently shrink 
at typical initial velocity of $-17 \mathrm{~km} \mathrm{~s}^{-1}$ and gradually decelerate to about $-5 \mathrm{~km} \mathrm{~s}^{-1}$ within $0.5-2.5 \mathrm{~h}$. They are all evidence of reconnection outflows during different stages (Figures [6] and 7).

3. The double coronal $X$-ray sources are spatially separated and located in the regions of bi-directional plasma outflows. The highest temperature is found near the loop-top X-ray source well below the reconnection site. This suggests that primary plasma heating and particle acceleration take place in the reconnection outflow regions (Holman 2012), rather than at the reconnection site itself. Models with this ingredient were proposed long ago (e.g., Forbes \& Priest 1983), but solid observational evidence as presented here has been lacking (Figures 10 and 11].

4. An energy dispersion is present in the loop-top position with higher-energy X-ray and hotter EUV emission located at greater heights over a large range of $\Delta h \geq 26 \mathrm{Mm}$. The $25-50 \mathrm{keV}$ nonthermal emission lies $15 \mathrm{Mm}$ (twice that of the Masuda flare) above the 6-10 keV thermal emission. This agrees with the expected trend of softer electron spectra in the nonthermal regime and lower temperatures in the thermal regime being associated with earlier energized loops, which are located further below the primary locus of energy release. The upper coronal X-ray source has an opposite trend because it is located in the oppositely directed reconnection outflow (Figures 3, 4, and 10).

5. Prior to the recently recognized descent followed by a continuous ascent, the overall loop-top emission experiences an initial ascent for nearly an hour. This is the first time that such motions, including the descent, are observed simultaneously from EUV to HXRs covering a wide range of temperatures of 1-30 MK. The transition from ascent to descent coincides with the rapid acceleration of the flux rope CME. The loop-top descends at $\sim 10 \mathrm{~km} \mathrm{~s}^{-1}$, about $50 \%$ slower in EUV than in X-ray (Figure 4).

6. The flare impulsive phase starts when a rapid velocity increases occur for the overall loop-top descent, the individual loop shrinkages, and the upward plasmoid ejections. This is delayed by 10 minutes from the initial loop-top descent, implying that the energy release rate is more intimately correlated with these velocities than the loop-top position (Figure 4).

\subsection{Proposed Physical Picture}

We propose the following physical picture to tie together the observations. The major points, including the location of particle acceleration and the three-stage (up-down-up) motion of the loop-top X-ray source, are sketched in Figure 12 .

The earlier confined $\mathrm{C} 4.5$ flare leads to the formation of a flux rope and cusp-shaped loops underneath it. After six hours of slow evolution, the flux rope becomes unstable and rises (Patsourakos et al. 2013). A vertical current sheet forms between two Y-type null points at the lower tip of the flux rope and the upper tip of the underlying cusp. Magnetic reconnection ensues within the current sheet, leading to the eruptive M7.7 flare.

Magnetic reconnection produces bi-directional outflows in forms of the observed plasmoids and contracting loops. Plasmoids are flux tubes formed by the tearing mode (Furth et al. 1963) with a guiding field along the current sheet. They are magnetic islands in two dimension or when the current sheet is seen edge-on. These outflows are driven by the magnetic tension force of the highly bent, newly reconnected field lines, as in those pointed cusps. The outflows generally decelerate, as observed here, when they run into the ambient corona and when the contracting loops relax to less bent shapes with reduced magnetic tension. This implies that the lowaltitude slow shrinkages could be the late stages of decelerated high-altitude fast contractions.

Several mechanisms can operate in the reconnection outflows and contribute to particle acceleration and plasma heating. Turbulence or plasma waves, for example, can be generated by the interaction of the high speed flows with the ambient corona. Upon cascading to smaller scales (comparable to the gyro-radii of background particles) at some distance from the reconnection site, turbulence can accelerate the particles and heat the plasma (Hamilton \& Petrosian 1992; Miller et al. 1996; Chandran 2003; Petrosian \& Liu 2004; Petrosian et al. 2006; Jiang et al. 2009; Fleishman \& Toptygin 2013). Additional contribution to particle acceleration and/or heating can come from fast-mode shocks in the outflow regions (Forbes \& Priest 1983; Tsuneta \& Naito 1998; Guo \& Giacalone 2012; Nishizuka \& Shibata 2013), gas dynamic shocks within contracting flux tubes (Longcope et al. 2009), and the first-order Fermi and betatron mechanisms within the collapsing traps formed by contracting loops (Somov \& Kosugi 1997; Karlický \& Kosugi 2004; Karlický 2006; Grady et al. 2012).

As the flux rope evolves into its fast rise and eruption stage, the current sheet could become sufficiently long causing significant tearing instability (e.g., Bárta et al. 2008), and/or become thinner than the ion skin depth leading to a transition from Sweet-Parker reconnection to collisionless Hall reconnection (Cassak et al. 2006). Both can result in enhanced rates of reconnection and energy release. The outflow velocity will also increase, producing stronger heating and particle acceleration by one or a combination of the above three mechanisms (turbulence, shock, and betatron) whose efficiency is expected to be positively correlated with the outflow velocity. For example, faster outflows can generate stronger turbulence that can lead to stronger particle acceleration (see Petrosian 2012, for a comparison between acceleration by turbulence and shocks). This explains the observed temporal correlation of the increase in outflow velocity and the onset of the impulse phase and HXR burst.

The loop-top source seen in X-ray and EUV represents the collective emission of the ensemble of flare loops, each of which undergoes post-reconnection contraction or shrinkage. The loop-top emission centroid is the average position of this ensemble, which in the thermal regime is determined by the convolution of the spatial distributions of temperature and emission measure. Such distributions are influenced by several competing processes, including energization of new loops produced by the $u p$ - 
(a)

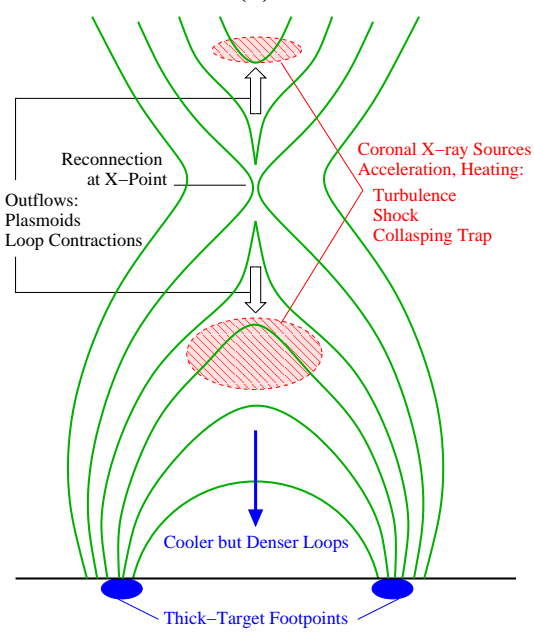

(b)

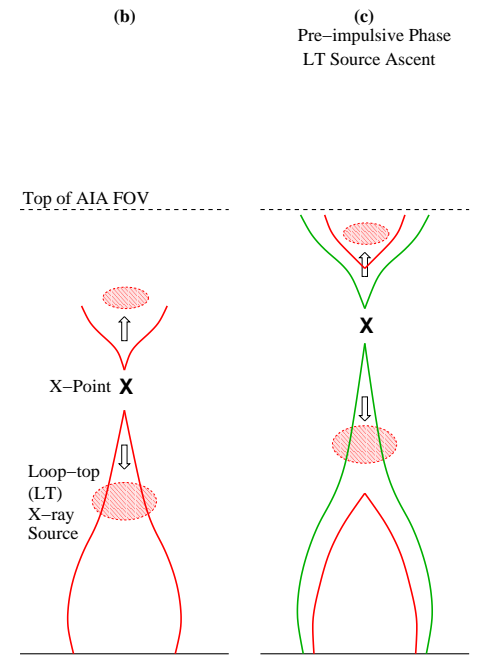

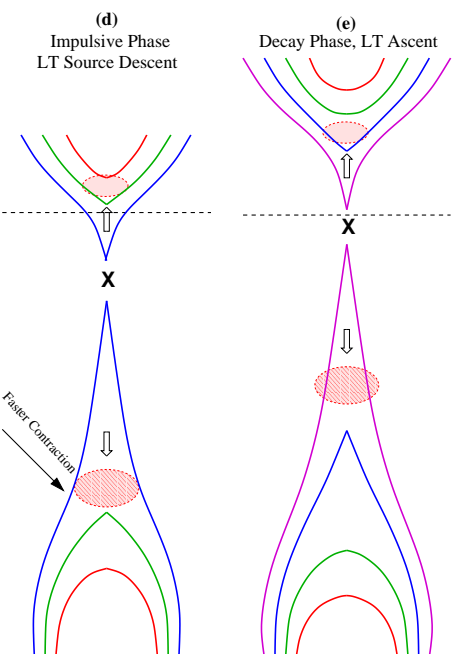

FIG. 12.- (a) Schematic of the proposed flare model in which particle acceleration and plasma heating take place in the reconnection outflow regions away from the reconnection site. (b)-(e) Temporal development of reconnection and the up-down-up three-stage motion of the loop-top (LT) X-ray source. We show only the portions of reconnected magnetic field lines near the reconnection site, above and below which each pair of field lines are identified by the same color during their relaxations. Note that the higher contraction speeds (e.g., of the green loop) from (c) to (d) is associated with the loop-top source descent. The upper coronal source is not hatched during the impulsive and decay phases, indicating its non-detection due to weak emission. We assume that bi-directional reconnection outflows and relaxations of field lines persist throughout the event, but the upward component is beyond the AIA FOV during the late phase and thus not observed.

ward developing reconnection, and downward contractions and cooling of previously reconnected loops. We suggest that the interplay of these processes results in the up-down-up migration of the loop-top source. Around the early impulsive phase, the observed higher velocities of loop contractions tend to shift the overall loop-top source downward, as depicted in Figures 12 (c) and (d). Within the contracting loops, conductive cooling can be considerably reduced by stronger turbulence produced by faster reconnection outflows (Chandran \& Cowlev 1998; Jiang et al. 2006). Slower cooling helps the X-ray/EUV emission of these hot loops last longer during their contractions and further contribute to the loop-top descent. Before and after the early impulsive phase, the situation could be different. Loop contractions are slower and associated faster cooling can make these loops cool below the instrumental temperature passband more rapidly. The upward development of reconnection could thus dominate, leading to the upward loop-top migration. This interpretation is supported by the observed temporal coincidence of the highest velocities of individual loop shrinkages and of the loop-top descent at the impulsive phase onset. Like cooling, chromospheric evaporation can also play a role. After the initial chromospheric evaporation associated with energization of newly reconnected loops, if it continues operating during the subsequent loop contractions, the densities and thus emission measures of these loops would keep rising and contribute to the downward loop-top drift. Otherwise, if it rapidly subsides, it would assist the upward loop-top drift. Investigating such a complex interplay would require detailed numerical modeling, which is beyond our scope here.

\subsection{Discussion}

The fast downward contractions of EUV loops are best seen during the decay phase, likely because of their greater distances traveled making them easier to be detected. Likewise, different environments may explain the factor of two difference in the median deceleration be- tween the early and later fast contractions (Figures 7 (f) and $(\mathrm{g}))$. The earlier, compact flare loops of stronger magnetic field can produce stronger resistance (e.g., by the Lorentz force from a reverse current; Bárta et al. 2008) to quickly brake the contracting loops impinging on them from above, while the later, large flare arcade of weaker field at greater heights and greater travel distances may allow more gradual deceleration.

The X-ray spectra are essentially thermal during the decay phase. This suggests that the late phase contractions are associated with plasma heating rather than particle acceleration. This is expected because the magnetic field strength decreases with height, and so does the available magnetic energy to be released by reconnection. Such prolonged heating may contribute to the wellknown slower than expected cooling of flare plasma (see Figure 1(d); McTiernan et al. 1993; Jiang et al. 2006).

The fast high-altitude contractions and slow lowaltitude shrinkages appear as two statistically distinct populations. The median final velocity of the former is more than four times the median initial velocity of the latter ( -73 vs. $-17 \mathrm{~km} \mathrm{~s}^{-1}$; Figures [6] and 7]). We have interpreted the latter as the late stages of the former that have decelerated, but we rarely see a continuous track decelerating from $>400 \mathrm{kms}^{-1}$ to a few $\mathrm{kms}^{-1}$. Alternatively, this distinction may be due to inhomogeneity of reconnection (see, e.g., Asai et al. 2004) in the current sheet above the flare arcade that is primarily oriented along the line of sight. The persistent slow shrinkages could result from reconnection at a moderate rate throughout the current sheet, which produces flare loops forming the arcade and double ribbons at their footpoints. The episodic fast contractions could be signatures of plasmoids within the current sheet, associated with enhanced reconnection and energy release rates. These fast contracting loops are dispersed along the line of sight amidst slowly shrinking loops, producing the observed effects (e.g., Figure $7(b)$ ).

There are other physically different (though not nec- 
essarily unrelated) phenomena that share similar observational signatures and should not be confused with the contractions of newly reconnected loops studied here. For example, pre-existing coronal loops can contract at typical speeds $<100 \mathrm{~km} \mathrm{~s}^{-1}$ during eruptions (Liu \& Wang 2010; Liu et al. 2012a; Sun et al. 2012), interpreted as implosion (Hudson 2000; Janse \& Low 2007) due to the rapid release of magnetic energy and thus reduction of magnetic pressure within the eruption volume. When a CME eruption initiates at an elevated height, it can produces a downward push to displace ambient coronal loops (e.g., Liu et al. 2012c, see their Figure 12(h), at $\left.-60 \mathrm{kms}^{-1}\right)$. Even slower shrinkage at $-3 \mathrm{kms}^{-1}$ can occur in active region loops due to gradual cooling (Wang et al. 1997). Sometimes, conjugate X-ray footpoints approach each other while the loop-top source descends (Ji et al. 2006; Liu et al. 2009a; Yang et al. 2009), likely because of reconnection progressing toward less sheared loops at lower altitudes. We have not found these signatures in the flare under study here, except for a few overlying existing loops that contract at about $-150 \mathrm{~km} \mathrm{~s}^{-1}$ during 05:40-05:50 UT near the end of the impulsive phase. In addition, some high-lying loops continue to expand and erupt at $\sim 100 \mathrm{kms}^{-1}$ up to 08:00 UT, three hours after the original flux rope eruption. Examination of such features and quantitative comparison of these observations with particle acceleration models will be subjects of future investigations.

W.L. was supported by NASA SDO/AIA contract NNG04EA00C and LWS/TR\&T contract NNX11AO68G. Q.R.C. and V.P. were supported by NASA grants NNX10AC06G and NNX13AF79G. W.L. thanks Marian Karlicky, Paul Cassak, Hugh Hudson, Sasha Kosovichev, Judy Karpen, Rick DeVore, Greg Slater, Tom Berger, and Mark Cheung for useful discussions.

\section{APPENDIX A}

\section{OBSERVATIONS OF ALL AIA CHANNELS}

In the main text, we focused on the $131 \AA$ channel. For a complete temperature coverage, we examine all AIA channels here. As shown in Figure 13 (top), each channel has a generally broad response with one to a few peaks. In the order of approximately decreasing temperature response are 193, 131, 94, 304, 335, 211, and $171 \AA$ channels.

Figure 13 shows space-time plots of all EUV channels. On the left are base ratio from every other frame of short exposure so that bright flare loops are not over-exposed, while on the right are running ratio from frames of regular exposure that is needed for detecting faint emission, including fast loop contractions, above the flare arcade. On the left, the 131 and $94 \AA$ channels capture the hot, diffuse loop-top emission, while the flare arcade underneath it is best seen in cooler channels. Both regions appear in the $193 \AA$ channel because of its dual response to hot Fe XXIV emission peaking at $18 \mathrm{MK}$ and cool Fe XII emission at 1.6 MK (O'Dwver et al. 2010). On the righthand side, downward fast contractions and slow shrink- ages can be identified in the three hottest channels. In cooler channels, cooling condensations rain down the arcade loops since 06:00 UT at up to $\sim 170 \mathrm{~km} \mathrm{~s}^{-1}$ or $90 \%$ of the free fall speed, faster than typical coronal rain (Antolin \& Rouppe van der Voort 2012).

In Figure 13 (left), the erupting flux rope is best seen in the hot 131 and $94 \AA$ channels as a bright, accelerating track, indicating its high temperature, as also shown in Figure 11. Its evolution from gradual rise to impulsive eruption is accompanied by the rapid increase in X-ray flux and the loop-top's ascent-to-descent transition. This is in line with observed synchronous eruption accelerations and flare onsets (Zhang et al. 2001, 2002; Temmer et al. 2008; Liu et al. 2011a).

Figures 1(b) and 4(a) show temporal emission profiles taken from the base-ratio space-time plots at $h_{\text {ref }}=60^{\prime \prime}$ marked by a cross. Since 04:17 UT, the 131 and $94 \AA$ emission increases considerably, while other emission remains essentially flat. This indicates gentle heating early in the flare, as seen in the temperature history at this position shown in Figure 11(j). The dip in all channels at the onset of the impulsive phase (vertical dashed line) is due to the descent of the loop-top source. The large hump afterwards results from the growth of the flare arcade toward greater heights. This hump generally progresses toward cooler channels, indicating a cooling sequence, similar to those recently observed in flares, AR loops, and prominence condensations (Woods et al. 2011; Viall \& Klimchuk 2012; Liu et al. 2012b; Berger et al. 2012). The double humps at $193 \AA$ are again due to its dual temperature response.

Like $193 \AA$, the $304 \AA$ channel responds to both hot Ca XVIII emission at 7.1 MK and cool He II emission at $0.05 \mathrm{MK}$. The dual response of these two channels leads to their unusually large descent of about $40 \%$ of the loop-top centroids (see Table 2). This is because their maximum loop-top heights are dominated by hot emission, while the minimum heights are dominated by cool emission at even lower heights (Figure 4).

\section{APPENDIX B}

DISAMBIGUATION WITH STEREO OBSERVATIONS

The limb view of $S D O /$ AIA leaves an alternative possibility of the loop-top descent as the progression of energization from high- to low-lying loops along the arcade in the east-west direction. This possibility is not supported by STEREO-A (STA) that was $121^{\circ}$ ahead of the Earth and observed the flare on the disk. When the X-ray loop-top descent occurs, the STA $195 \AA$ image (Figure [14(d)) shows a compact cusp-shaped loop, while the arcade system develops later (panels (e) and (f)). One could further argue that a temperature effect, i.e., cooling or heating, can make a progression appear as a compact source rather than an arcade. If so, the loops being cooled or heated would have appeared at the same location and been captured by AIA in cooler or hotter EUV channels or by RHESSI in X-rays. Instead, all available EUV and X-ray data covering a wide temperature range of $10^{5}-10^{7} \mathrm{~K}$ show a consistent loop-top descent, which must be a real mass motion.

\section{REFERENCES}

Antolin, P. \& Rouppe van der Voort, L. 2012, ApJ, 745, 152
Asai, A., Yokoyama, T., Shimojo, M., \& Shibata, K. 2004, ApJ, $605, \mathrm{~L} 77$ 

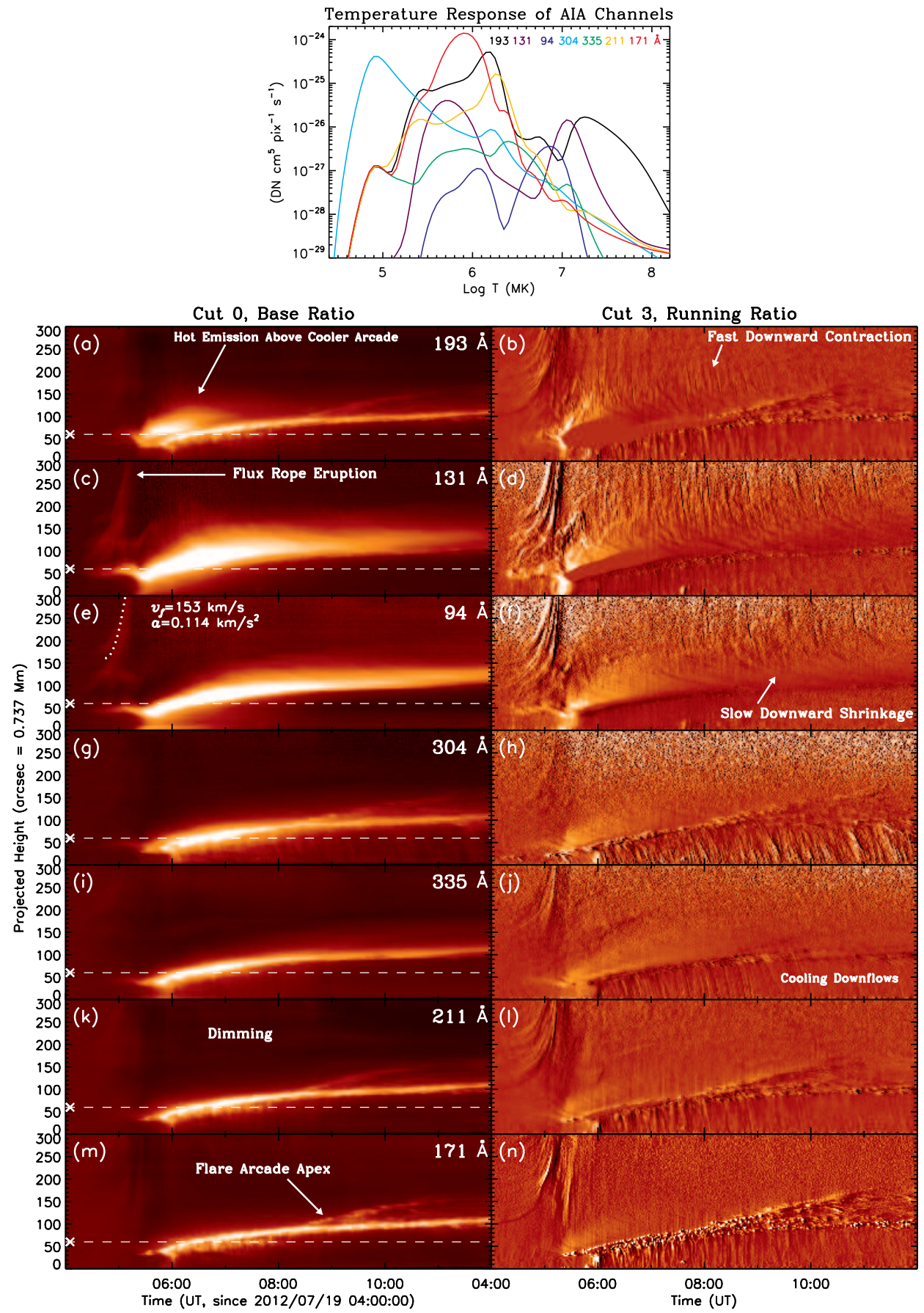

Fig. 13. - Top: Temperature response of AIA EUV channels. Other panels: Space-time plots of all AIA EUV channels in base ratio from Cut 0 on the left and in running ratio from Cut 3 on the right. The panels are arranged approximately in the order from high to low temperature response. The cross and horizontal dash line on the left mark the slice for obtaining the temporal profiles shown in Figure 1 b). The dotted line in (e) is a parabolic fit to the central portion of the erupting flux rope CME that has an average acceleration of $a=0.114 \pm 0.002 \mathrm{~km} \mathrm{~s}^{-2}$ and achieves a velocity of $v_{f}=153 \pm 3 \mathrm{~km} \mathrm{~s}^{-1}$ at the edge of AIA's FOV.

Aschwanden, M. J., Boerner, P., Schrijver, C. J., \& Malanushenko, A. 2011, Sol. Phys., 384

Bain, H. M., Krucker, S., Glesener, L., \& Lin, R. P. 2012, ApJ, 750,44

Bárta, M., Vršnak, B., \& Karlický, M. 2008, A\&A, 477, 649

Battaglia, M., Fletcher, L., \& Benz, A. O. 2009, A\&A, 498, 891

Battaglia, M. \& Kontar, E. P. 2012, ApJ, 760, 142

Berger, T. E., Liu, W., \& Low, B. C. 2012, ApJ, 758, L37

Carmichael, H. 1964, in The Physics of Solar Flares, ed. W. N. Hess, 451

Caspi, A. \& Lin, R. P. 2010, ApJ, 725, L161

Cassak, P. A. \& Drake, J. F. 2013, submitted to ApJ
Cassak, P. A., Drake, J. F., \& Shay, M. A. 2006, ApJ, 644, L145 Chandran, B. D. G. 2003, ApJ, 599, 1426

Chandran, B. D. G. \& Cowley, S. C. 1998, Physical Review Letters, 80, 3077

Chen, Q. \& Petrosian, V. 2012, ApJ, 748, 33

Fleishman, G. D. \& Toptygin, I. N. 2013, MNRAS, 447

Fletcher, L., Dennis, B. R., Hudson, H. S., et al. 2011, Space Sci. Rev., 159, 19

Forbes, T. G. \& Acton, L. W. 1996, ApJ, 459, 330

Forbes, T. G. \& Priest, E. R. 1983, Sol. Phys., 84, 169

Furth, H. P., Killeen, J., \& Rosenbluth, M. N. 1963, Physics of Fluids, 6, 459 


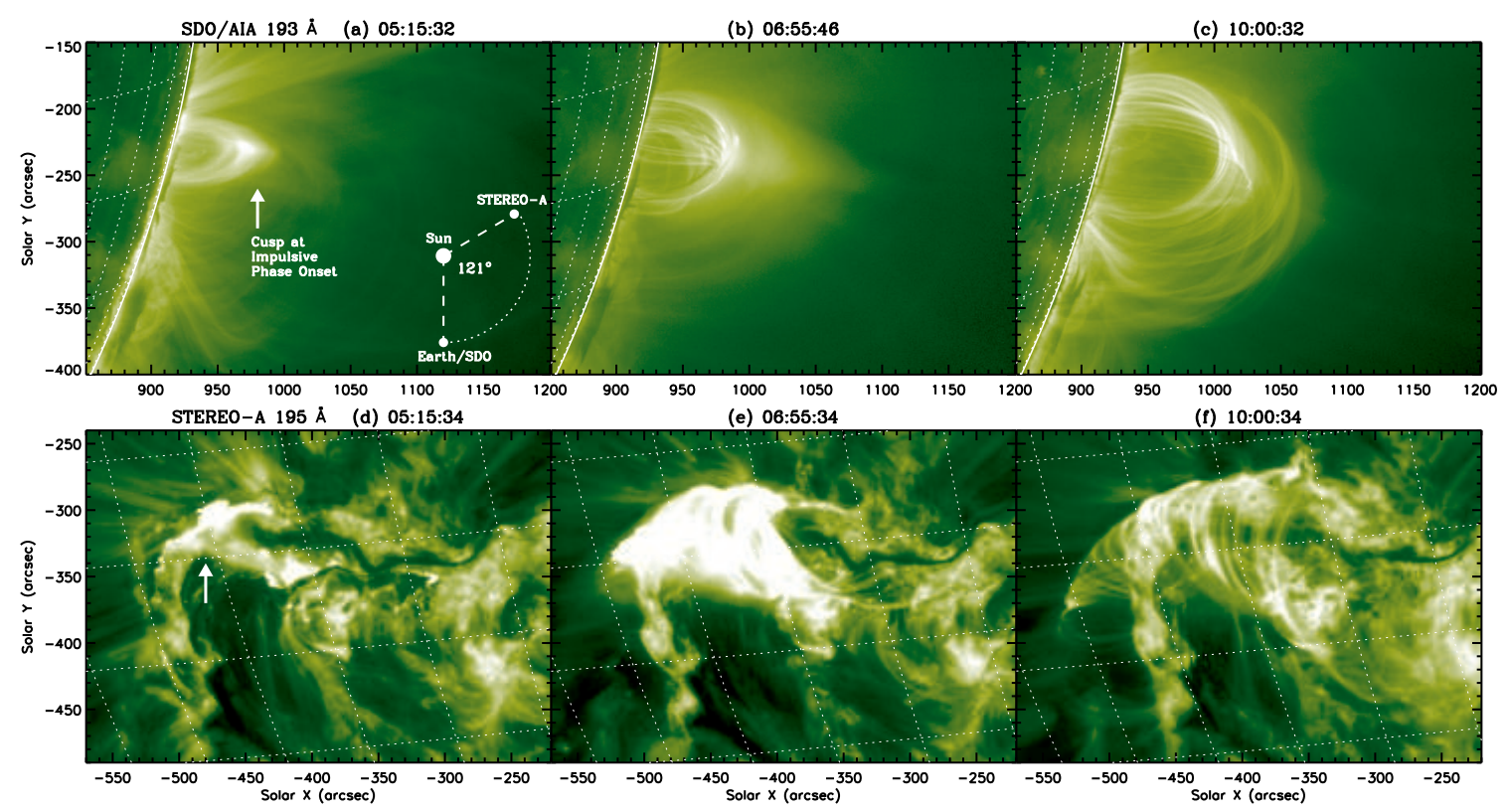

FIG. 14.- Comparison of SDO/AIA $193 \AA$ (top) and STEREO-A $195 \AA$ (bottom) images of the flare. The STEREO images are scaled as if they are viewed from the same heliocentric distance as $S D O$. The heliographic grids are spaced at $5^{\circ}$. Note the compact brightening at the impulsive phase onset (pointed by the arrow), in contrast to the extended arcade system later.

Glesener, L., Krucker, S., \& Lin, R. P. 2012, ApJ, 754, 9

Grady, K. J., Neukirch, T., \& Giuliani, P. 2012, A\&A, 546, A85

Guo, F. \& Giacalone, J. 2012, ApJ, 753, 28

Haerendel, G. 2009, ApJ, 707, 903

Hamilton, R. J. \& Petrosian, V. 1992, ApJ, 398, 350

Hara, H., Watanabe, T., Harra, L. K., Culhane, J. L., \& Young, P. R. 2011, ApJ, 741, 107

Hirayama, T. 1974, Sol. Phys., 34, 323

Holman, G. D. 2012, Physics Today, 65, 56

Holman, G. D., Aschwanden, M. J., Aurass, H., Battaglia, M., Grigis, P. C., Kontar, E. P., Liu, W., Saint-Hilaire, P., \& Zharkova, V. V. 2011, Space Sci. Rev., 159, 107

Hudson, H. S. 2000, ApJ, 531, L75

Innes, D. E., Inhester, B., Axford, W. I., \& Wilhelm, K. 1997, Nature, 386, 811

Innes, D. E., McKenzie, D. E., \& Wang, T. 2003, Sol. Phys., 217, 247

Janse, Å. M. \& Low, B. C. 2007, A\&A, 472, 957

Ji, H., Huang, G., Wang, H., Zhou, T., Li, Y., Zhang, Y., \& Song, M. 2006, ApJ, 636, L173

Jiang, Y. W., Liu, S., Liu, W., \& Petrosian, V. 2006, ApJ, 638, 1140

Jiang, Y. W., Liu, S., \& Petrosian, V. 2009, ApJ, 698, 163

Karlický, M. 2006, Space Sci. Rev., 122, 161

Karlický, M. \& Kosugi, T. 2004, A\&A, 419, 1159

Karpen, J. T., Antiochos, S. K., \& DeVore, C. R. 2012, ApJ, 760, 81

Khan, J. I., Bain, H. M., \& Fletcher, L. 2007, A\&A, 475, 333

Ko, Y.-K., Raymond, J. C., Lin, J., Lawrence, G., Li, J., \& Fludra, A. 2003, ApJ, 594, 1068

Kopp, R. A. \& Pneuman, G. W. 1976, Sol. Phys., 50, 85

Krucker, S., Hudson, H. S., Glesener, L., White, S. M., Masuda,

S., Wuelser, J.-P., \& Lin, R. P. 2010, ApJ, 714, 1108

Lemen, J. R., Title, A. M., Akin, D. J., et al. 2012, Sol. Phys. 275, 17

Li, Y. P. \& Gan, W. Q. 2005, ApJ, 629, L137

—. 2006, ApJ, 644, L97

-. 2007, Advances in Space Research, 39, 1389

Lin, J. 2004, Sol. Phys., 222, 115

Lin, J., Ko, Y.-K., Sui, L., Raymond, J. C., Stenborg, G. A. Jiang, Y., Zhao, S., \& Mancuso, S. 2005, ApJ, 622, 1251

Lin, R. P., Dennis, B. R., Hurford, G. J., et al. 2002, Sol. Phys., 210,3

Liu, R. 2013, submitted to ApJ

Liu, R., Lee, J., Wang, T., Stenborg, G., Liu, C., \& Wang, H. 2010, ApJ, 723, L28
Liu, R., Liu, C., Török, T., Wang, Y., \& Wang, H. 2012a, ApJ, 757,150

Liu, R. \& Wang, H. 2010, ApJ, 714, L41

Liu, W., Berger, T. E., \& Low, B. C. 2012b, ApJ, 745, L21

Liu, W., Berger, T. E., Title, A. M., Tarbell, T. D., \& Low, B. C. 2011a, ApJ, 728, 103

Liu, W., Jiang, Y. W., Liu, S., \& Petrosian, V. 2004, ApJ, 611, L53

Liu, W., Ofman, L., Nitta, N. V., Aschwanden, M. J., Schrijver, C. J., Title, A. M., \& Tarbell, T. D. 2012c, ApJ, 753, 52

Liu, W., Petrosian, V., Dennis, B. R., \& Holman, G. D. 2009a, ApJ, 693, 847

Liu, W., Petrosian, V., Dennis, B. R., \& Jiang, Y. W. 2008, ApJ, 676, 704

Liu, W., Petrosian, V., \& Mariska, J. T. 2009b, ApJ, 702, 1553

Liu, W., Title, A. M., Zhao, J., Ofman, L., Schrijver, C. J., Aschwanden, M. J., De Pontieu, B., \& Tarbell, T. D. 2011b, ApJ, 736, L13

Liu, W., Wang, T.-J., Dennis, B. R., \& Holman, G. D. 2009c, ApJ, 698, 632

Longcope, D. W., Guidoni, S. E., \& Linton, M. G. 2009, ApJ, 690, L18

Masuda, S., Kosugi, T., Hara, H., Tsuneta, S., \& Ogawara, Y. 1994, Nature, 371, 495

McKenzie, D. E. \& Hudson, H. S. 1999, ApJ, 519, L93

McTiernan, J. M., Kane, S. R., Loran, J. M., Lemen, J. R., Acton, L. W., Hara, H., Tsuneta, S., \& Kosugi, T. 1993, ApJ, 416, L91

Miller, J. A., Larosa, T. N., \& Moore, R. L. 1996, ApJ, 461, 445

Milligan, R. O. \& Dennis, B. R. 2009, ApJ, 699, 968

Milligan, R. O., McAteer, R. T. J., Dennis, B. R., \& Young, C. A. 2010, ApJ, 713, 1292

Murphy, N. A., Miralles, M. P., Pope, C. L., et al. 2012, ApJ, 751,56

Nakariakov, V. M. \& Melnikov, V. F. 2009, Space Sci. Rev., 149, 119

Neupert, W. M. 1968, ApJ, 153, L59

Nishizuka, N. \& Shibata, K. 2013, ArXiv e-prints

Nishizuka, N., Takasaki, H., Asai, A., \& Shibata, K. 2010, ApJ, 711,1062

Nitta, N. V., Freeland, S. L., \& Liu, W. 2010, ApJ, 725, L28

O'Dwyer, B., Del Zanna, G., Mason, H. E., Weber, M. A., \& Tripathi, D. 2010, A\&A, 521, A21

Ofman, L., Liu, W., Title, A., \& Aschwanden, M. 2011, ApJ, 740, L33

Patsourakos, S., Vourlidas, A., \& Stenborg, G. 2013, ApJ, 764, 125 
Petrosian, V. 2012, Space Sci. Rev., 173, 535

Petrosian, V. \& Liu, S. 2004, ApJ, 610, 550

Petrosian, V., Yan, H., \& Lazarian, A. 2006, ApJ, 644, 603

Pick, M., Démoulin, P., Krucker, S., Malandraki, O., \& Maia, D. 2005, ApJ, 625, 1019

Raymond, J. C., Krucker, S., Lin, R. P., \& Petrosian, V. 2012 , Space Sci. Rev., 173, 197

Reeves, K. K., Seaton, D. B., \& Forbes, T. G. 2008, ApJ, 675, 868

Reznikova, V. E., Melnikov, V. F., Ji, H., \& Shibasaki, K. 2010, ApJ, 724, 171

Savage, S. L., Holman, G., Reeves, K. K., Seaton, D. B., McKenzie, D. E., \& Su, Y. 2012, ApJ, 754, 13

Savage, S. L. \& McKenzie, D. E. 2011, ApJ, 730, 98

Sheeley, Jr., N. R., Warren, H. P., \& Wang, Y.-M. 2004, ApJ, 616,1224

Shen, C., Lin, J., \& Murphy, N. A. 2011, ApJ, 737, 14

Shen, J., Zhou, T., Ji, H., Wang, N., Cao, W., \& Wang, H. 2008, ApJ, 686, L37

Shen, Y. \& Liu, Y. 2012, ApJ, 753, 53

Somov, B. V. \& Kosugi, T. 1997, ApJ, 485, 859

Sturrock, P. A. 1966, Nature, 211, 695

Su, Y., Dennis, B. R., Holman, G. D., Wang, T., Chamberlin, P. C., Savage, S., \& Veronig, A. 2012, ApJ, 746, L5

Sui, L. \& Holman, G. D. 2003, ApJ, 596, L251

Sui, L., Holman, G. D., \& Dennis, B. R. 2004, ApJ, 612, 546

Sun, X., Hoeksema, J. T., Liu, Y., Wiegelmann, T., Hayashi, K., Chen, Q., \& Thalmann, J. 2012, ApJ, 748, 77

Švestka, Z., Fárník, F., Hudson, H. S., \& Hick, P. 1998, Sol. Phys., 182, 179

Švestka, Z. F., Fontenla, J. M., Machado, M. E., Martin, S. F., \& Neidig, D. F. 1987, Sol. Phys., 108, 237
Takasao, S., Asai, A., Isobe, H., \& Shibata, K. 2012, ApJ, 745, L6 Tandberg-Hanssen, E. \& Emslie, A. G. 1988, The physics of solar flares (Cambridge and New York: Cambridge University Press, 286 p.)

Temmer, M., Veronig, A. M., Vršnak, B., Rybák, J., Gömöry, P., Stoiser, S., \& Maričić, D. 2008, ApJ, 673, L95

Tsuneta, S. \& Naito, T. 1998, ApJ, 495, L67

Veronig, A. M., Karlický, M., Vršnak, B., Temmer, M., Magdalenić, J., Dennis, B. R., Otruba, W., \& Pötzi, W. 2006, A\&A, 446, 675

Viall, N. M. \& Klimchuk, J. A. 2012, ApJ, 753, 35

Vršnak, B., Temmer, M., Veronig, A., Karlický, M., \& Lin, J. 2006, Sol. Phys., 234, 273

Wang, J., Shibata, K., Nitta, N., Slater, G. L., Savy, S. K., \& Ogawara, Y. 1997, ApJ, 478, L41

Wang, T. J., Sui, L., \& Qiu, J. 2007, ApJ, 661, L207

Wang, Y.-M., Sheeley, N. R., Howard, R. A., St. Cyr, O. C., \& Simnett, G. M. 1999, Geophys. Res. Lett., 26, 1203

Warren, H. P., O'Brien, C. M., \& Sheeley, Jr., N. R. 2011, ApJ, 742,92

Watanabe, T., Hara, H., Sterling, A. C., \& Harra, L. K. 2012, Sol. Phys., 185

Woods, T. N., Hock, R., Eparvier, F., et al. 2011, ApJ, 739, 59 Yang, Y.-H., Cheng, C. Z., Krucker, S., Lin, R. P., \& Ip, W. H. 2009, ApJ, 693, 132

Yokoyama, T., Akita, K., Morimoto, T., Inoue, K., \& Newmark, J. 2001, ApJ, 546, L69

Zhang, J., Dere, K. P., Howard, R. A., Kundu, M. R., \& White, S. M. 2001, ApJ, 559, 452

Zhang, M., Golub, L., DeLuca, E., \& Burkepile, J. 2002, ApJ, 574, L97 